\title{
JNK1 determines the oncogenic or tumor- suppressive activity of the integrin-linked kinase in human rhabdomyosarcoma
}

\author{
Adam D. Durbin,, ${ }^{1,2,3}$ Gino R. Somers, ${ }^{4,5}$ Michael Forrester, ${ }^{3}$ Malgorzata Pienkowska, ${ }^{3}$ \\ Gregory E. Hannigan, ${ }^{6}$ and David Malkin ${ }^{1,3}$
}

\begin{abstract}
${ }^{1}$ Department of Medical Biophysics and 2MD/PhD Program, University of Toronto, Toronto, Ontario, Canada. ${ }^{3}$ Program in Genetics and Genome Biology, Hospital for Sick Children, Toronto, Ontario, Canada. ${ }^{4 D}$ epartment of Laboratory Medicine and Pathobiology, University of Toronto, Toronto, Ontario, Canada.

${ }^{5}$ Department of Pathology, Hospital for Sick Children, Toronto, Ontario, Canada. ${ }^{6}$ Monash Institute of Medical Research, Melbourne, Victoria, Australia.
\end{abstract}

\begin{abstract}
Although most reports describe the protein kinase integrin-linked kinase (ILK) as a proto-oncogene, occasional studies detail opposing functions in the regulation of normal and transformed cell proliferation, differentiation, and apoptosis. Here, we demonstrated that ILK functions as an oncogene in the highly aggressive pediatric sarcoma alveolar rhabdomyosarcoma (ARMS) and as a tumor suppressor in the related embryonal rhabdomyosarcoma (ERMS). These opposing functions hinge on signaling through a noncanonical ILK target, JNK1, to the proto-oncogene c-Jun. RNAi-mediated depletion of ILK induced activation of JNK and its target, c-Jun, resulting in growth of ERMS cells, whereas in ARMS cells, it led to loss of JNK/c-Jun signaling and suppression of growth both in vitro and in vivo. Ectopic expression of the fusion gene characteristic of ARMS (paired box 3-forkhead homolog in rhabdomyosarcoma [PAX3-FKHR]) in ERMS cells was sufficient to convert them to an ARMS signaling phenotype and render ILK activity oncogenic. Furthermore, restoration of JNK1 in ARMS reestablished a tumor-suppressive function for ILK. These findings indicate what we believe to be a novel effector pathway regulated by ILK, provide a mechanism for interconversion of oncogenic and tumor-suppressor functions of a single regulatory protein based on the genetic background of the tumor cells, and suggest a rationale for tailored therapy of rhabdomyosarcoma based on the different activities of ILK.
\end{abstract}

\section{Introduction}

Rhabdomyosarcoma (RMS) is the most common soft tissue sarcoma of childhood. Despite advances in multimodality therapy, the failure-free survival of patients diagnosed with metastatic RMS remains low, at 25\% (1). RMS tumors are believed to arise from committed mesenchymal or myogenic progenitor cells that accumulate genetic damage and fail to terminally differentiate (2). RMS tumors largely belong to 2 different histologic subgroupings: $63 \%$ are of embryonal RMS (ERMS) histology, while nearly $20 \%$ are of alveolar RMS (ARMS) histology (2). In addition to the morphologic and ultrastructural differences between these 2 tumor histologies, they display distinct clinical profiles. ARMS tumors are more likely to be found in the extremities of older children, while ERMS tumors are more commonly found in the head and neck, trunk, and genitourinary systems of younger children (2). ARMS tumors are further associated with a poorer clinical course, exemplified by a higher incidence of bone marrow and lung metastases and higher mortality rate (2).

The molecular differences between ARMS and ERMS tumors have been intensively studied (2). Of ARMS tumors, 70\% display chromosomal translocations, fusing the neuromuscular development-associated genes paired box $3(P A X 3)$ and $P A X 7$ to the PKB/

Conflict of interest: The authors have declared that no conflict of interest exists. Nonstandard abbreviations used: AP-1, activator protein 1; ARMS, alveolar RMS; ASK1, apoptosis signal-regulating kinase 1; ERMS, embryonal RMS; FKHR, forkhead homolog in rhabdomyosarcoma; GSK3 $\beta$, glycogen synthase kinase- $3 \beta$; ILK, integrin-linked kinase; LOH, loss of heterozygosity; MTT, 3-(4,5-dimethylthiazol-2yl)-2,5-diphenyltetrazolium bromide; PAX3, paired box 3; RMS, rhabdomyosarcoma; TAM67, c-Jun transactivation domain mutant.

Citation for this article: J. Clin. Invest. 119:1558-1570 (2009). doi:10.1172/JCI37958.
Akt-regulated transcription factor forkhead in rhabdomyosarco$\mathrm{ma}$ (FKHR; also referred to as forkhead box O1A). These translocations occur in frame, yielding chimeric transcription factors that are poor prognostic indicators in the diagnosis of ARMS (2). The introduction of PAX-FKHR fusions into ERMS cells results in dramatic alterations in signaling pathways, including upregulation of JNK/MAPK signaling pathway members apoptosis signal-regulating kinase 1 (ASK1), hemopoietic progenitor kinase (HPK), and c-Jun (3). ERMS tumors commonly display loss of heterozygosity $(\mathrm{LOH})$ or loss of imprinting through genomic lesions at chromosome 11p15.4/5 (2). Intriguingly, $\mathrm{LOH}$ in a region spanning the microsatellite markers D11S1323 to D11S1338 at $11 \mathrm{p} 15.4 / 5$ correlated with invasive and metastatic disease in primary breast carcinomas, and the markers flanked a single annotated gene, integrinlinked kinase (ILK; ref. 4).

ILK was initially described as a $\beta_{1}$-integrin subunit-binding protein involved in kinase signaling pathways regulating growth and survival (5). ILK contains a kinase domain involved in the phosphorylation of numerous other pro-oncogenic kinases, including PKB/Akt, glycogen synthase kinase- $3 \beta$ (GSK3 $\beta$ ), and members of the MAPK signaling pathway (reviewed in ref. 6). Elevated ILK expression correlates with poor prognosis in multiple primary tumors, including melanoma and prostate, non-small cell lung, colorectal, ovarian, and anaplastic thyroid carcinomas (reviewed in ref. 6). Overexpression of ILK driven by mouse mammary tumor virus results in breast ductal hyperplasias and mammary tumors in transgenic mice (7). Furthermore, ILK inhibition using a novel small molecule has been previously demonstrated to be effective in preclinical xenograft models of glioblastoma and pancreatic and prostatic carcinomas (8-10). 
In contrast, some studies have suggested a variant growth-suppressive function for ILK in certain cellular contexts. Keratinocyterestricted knockout of ILK leads to induction of proliferation (11). Furthermore, a dominant-negative ILK mutant, E359K, blocks cell cycle exit and differentiation of L6 rat myoblasts (12). Similarly, ILK expression correlates in many tumor and normal tissues with areas of increased differentiation (13) and can cooperate with ionizing radiation to induce apoptosis in some cancer cells (14-16). Finally, overexpression of wild-type, but not mutant, ILK could limit tumor formation in MDA-MB-435 xenografts (17), although the mechanism for this finding was not reported.

The JNK family of kinases regulates transcription through phosphorylation and activation of a diverse set of transcription factor targets, including the proto-oncogene c-Jun, in response to both pro- and antiapoptotic stimuli (18). Phosphorylation on Ser63 of c-Jun by activated JNKs results in changes in transcriptional output and is associated with induction of cell cycle progression, upregulation of angiogenic survival and proliferation gene expression, and downregulation of death signaling through the Fas/Fas ligand extrinsic death receptor pathways (18). JNK activity is induced downstream of the MAPKKK ASK1 $(19,20)$, and both JNK and c-Jun are implicated in the repression of differentiation of RMS and maintenance of a tumorigenic state (21-24). ILK is also known to cross-talk with the JNK pathway and induce growth by potentiating the nuclear entry of the c-Jun coactivator nascent polypeptide-associated complex and coactivator $\alpha$ (25), as well as through the removal of inhibitory phosphorylations on ASK1 by the ILK-binding phosphatase ILK-associated serine/threonine phosphatase 2C (26).

Here, we investigate the hypothesis that ILK functions differently in ERMS and ARMS cells. We found that ILK was pro-oncogenic in ARMS and tumor suppressive in ERMS in vitro and in vivo. The tumor-suppressive function of ILK relied on the regulation of JNK1: depletion of ILK led to activation of JNK/c-Jun signaling in ERMS cells that was responsible for growth. ARMS cells, in contrast, failed to activate JNK1 and c-Jun in the absence of ILK, which resulted in reduced cell growth. We further demonstrated that introducing PAX3-FKHR into ERMS cells downregulated JNK1 and converted ERMS JNK/c-Jun signaling to ARMS oncogenic signaling. Finally, restoration of JNK1 in ARMS cells converted ILK from an oncogene into a tumor suppressor. These findings implicate a JNK/c-Jun signaling pathway in the regulation of cell fate by ILK and suggest that ILK may represent a target of therapeutic inhibition in ARMS, while exploration of JNK pathway inhibitors may be warranted in metastatic ERMS tumors.

\section{Results}

ILK is expressed and active in RMS and correlates with survival in ERMS. To examine the role of ILK in RMS, we first measured the levels of ILK expression in cell culture and primary tumors. We detected expression of ILK at the mRNA and protein levels in all ERMS and ARMS cell lines (Figure 1, A and B). Relative expression of ILK mRNA and protein did not correlate in A204 and Rh18 cells, suggesting posttranslational regulation of ILK, as previously reported by Aoyagi et al. (27). We next established the functionality of ILK in RMS cell lines by performing in vitro kinase assays. We observed similar levels of specific activity of ILK across all RMS cell lines, regardless of histology (Figure 1C). These data are consistent with prior reports of ILK expression in vitro (28), but not in vivo (29). To address this apparent discrepancy between the in vitro and in vivo environments, we examined ILK mRNA expression in primary RMS tumors. ILK mRNA expression was consistently low in ARMS samples, regardless of tumor stage. Similar to the cell lines, ILK mRNA expression was higher in ERMS than ARMS tumors. Intriguingly, however, lower levels were detected in high-stage ERMS samples (Figure 1D). Next, we determined the expression of ILK in primary RMS tumors by immunohistochemistry on a panel of tissue microarrays and whole-tumor slides, where, similar to the mRNA studies, higher ILK protein expression was detected in ERMS compared with ARMS tumors (Figure 1, E and F). Furthermore, subdivision of ERMS tumors by stage demonstrated reduced ILK protein levels in high-stage tumors (Figure 1G). Similarly, subdivision of ERMS tumors by ILK immunohistochemical score demonstrated that reduced ILK stain correlated with reduced overall patient survival, but not relapse-free survival (Figure 1, H and I).

ILK plays contradictory roles in vitro. These data indicated that expression levels of ILK are reduced in high-stage, poor-prognosis ERMS and correlate with increased survival, suggesting that ILK may play a role as a tumor suppressor in ERMS tumors. To directly assess the function of ILK in RMS, we knocked down and overexpressed ILK by performing transient siRNA and adenoviral infection of wild-type and kinase-inactive (R211A; ref. 30) ILK, respectively (Figure $2 \mathrm{~A}$ ). ILK kinase activity was reduced in RD ERMS and Rh4 ARMS cells after ILK-targeted RNAi and was induced with overexpression of wild type ILK, but not ILK-R211A (Figure 2, B and C). Intriguingly, overexpression of ILK induced soft agar colony formation and proliferation in 3 ARMS lines, but reduced these behaviors in 3 ERMS cell lines (Figure 2, D and E). In contrast, overexpression of kinase-inactive ILK-R211A had no effect in ERMS or ARMS cells (Figure 2, D and E). Furthermore, RNAi-mediated silencing of ILK with single or pooled siRNAs resulted in suppression of proliferation, clonogenicity, and soft agar colony formation in 3 ARMS cell lines, but stimulated these activities in 3 ERMS cell lines (Figure 2, F and G, and Supplemental Figure 1; supplemental material available online with this article; doi:10.1172/JCI37958DS1). These data suggested opposing roles for ILK in ERMS and ARMS cells. Since a prior hypothesis suggested that RMS arises from mesenchymal progenitor cells (31), and ERMS and ARMS cells displayed a strikingly opposite function for ILK, we sought to determine the function of ILK in primary mesenchymal progenitor cells. Similar to ERMS cells, overexpression of wild-type ILK, but not ILK-R211A, in primary human mesenchymal progenitor cells led to reductions of proliferation and clonogenicity (Supplemental Figure 2). Taken together, these data suggest that ILK plays a tumor-suppressive role in ERMS and mesenchymal progenitors in vitro in a kinase-dependent manner, but stimulates growth in ARMS cells, also in a kinase-dependent manner. To determine whether these in vitro effects occurred in vivo, we examined the role of ILK in mediating growth in murine models of RMS.

ILK both promotes and represses growth in murine xenografts. To assess the role of ILK in vivo, we subcutaneously xenografted female Balb/c nu/nu mice with RD (ERMS) or Rh4 (ARMS) cells treated with nothing or with $I L K$ or control siRNAs. Analysis demonstrated that ILK silencing induced rapid growth of ERMS xenografts, resulting in a doubling of tumor volume at day 2 after implantation $(P<0.05$ from day 2 until sacrifice, ANOVA; Figure 3, A and C). Furthermore, ILK-depleted, but not control, ERMS xenografts demonstrated local invasion and distant metastases (Table 1). In contrast, ILK silencing in ARMS xenografts resulted 
A

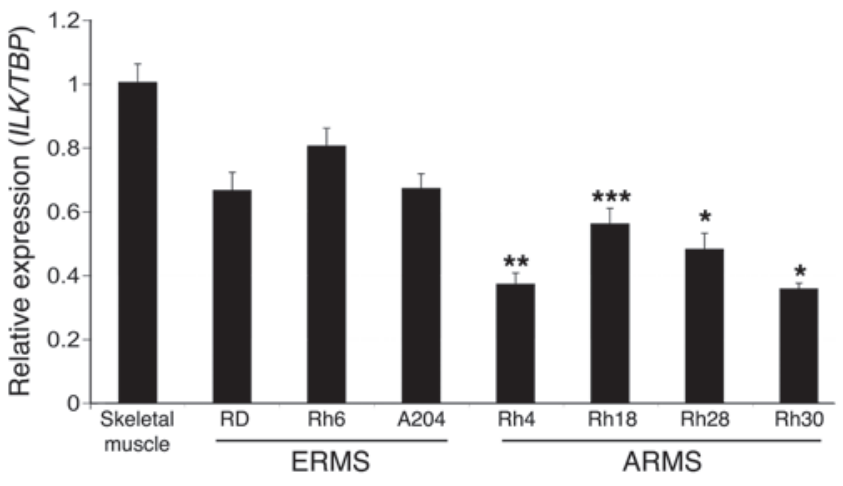

D

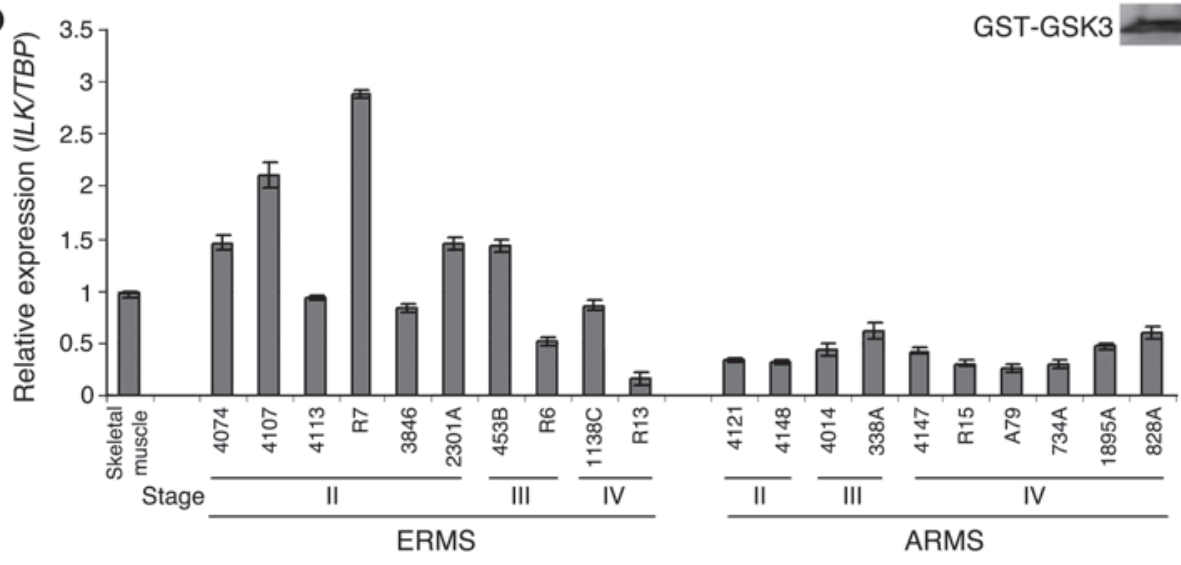

B
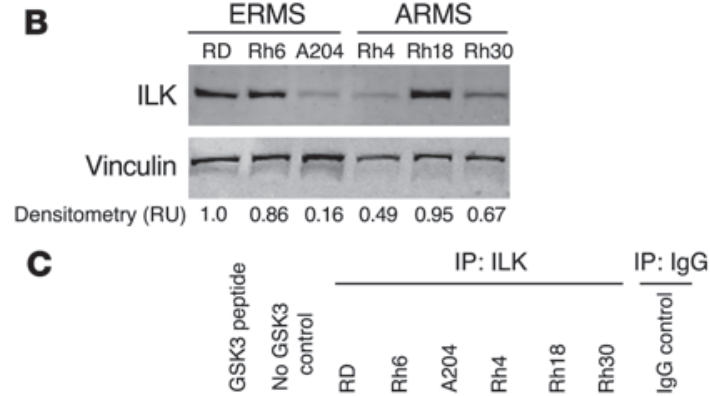

phospho-GSK3

ILK

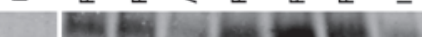

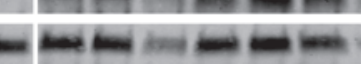

E

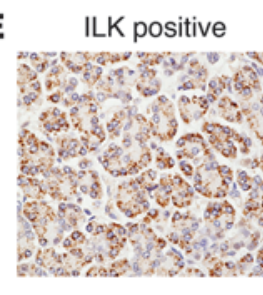

G

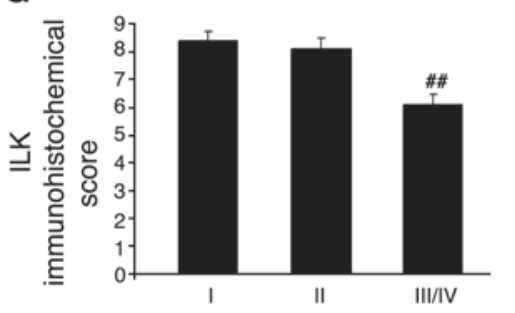

ILK negative

ERMS
ARMS

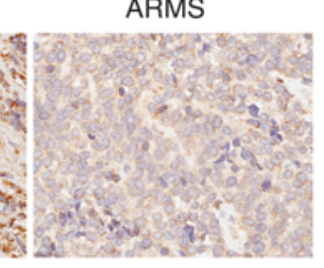

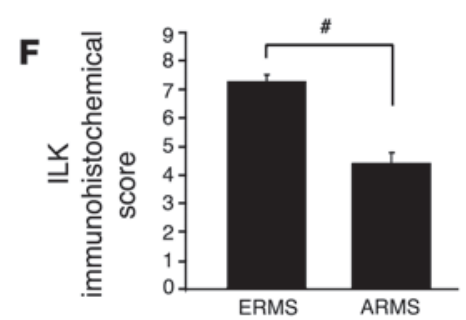
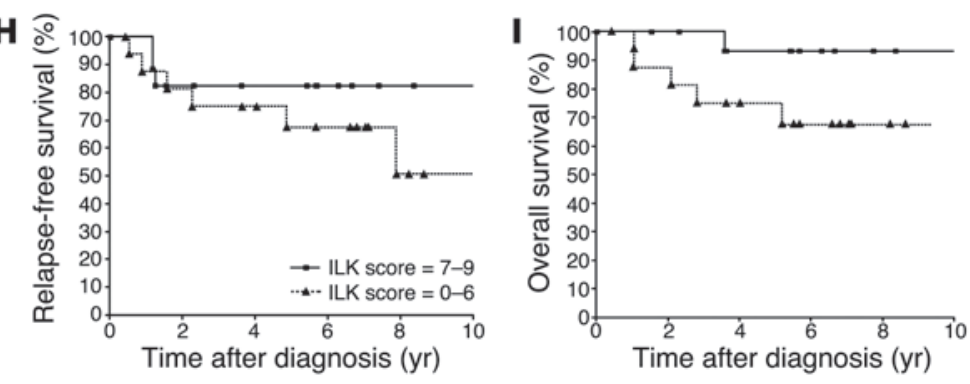

Figure 1

ILK expression and activity in RMS and tumor progression. (A) Quantitative RT-PCR profiling of ILK mRNA in RMS cell lines ( $n=4)$. Results were normalized to a skeletal muscle RNA pool. ${ }^{\star} P<0.05$, ${ }^{\star \star} P<0.01,{ }^{\star \star \star} P<0.001$ versus ERMS and skeletal muscle control, ANOVA. (B) RMS cell lysates were Western blotted for relative ILK expression. Data are representative of 3 independent trials. (C) Comparative ILK in vitro kinase assay in RMS cell lines. Data are representative of 4 independent trials. Lanes were run on the same gel but were noncontiguous (white line). (D) ILK mRNA levels were assessed in RMS primary tumors by quantitative RT-PCR $(n=3)$. Results were normalized to a skeletal muscle RNA pool. (E) Representative ILK immunohistochemistry in RMS tumors. Normal pancreas is shown as a positive and negative (primary antibody omitted) control. Original magnification, $\times 40$. (F) ILK immunohistochemical scoring of ARMS $(n=43)$ and ERMS $(n=52)$ tumors. $\# P=3.41 \times 10^{-9}$, Student's $t$ test. (G) ILK immunohistochemical score in ERMS tumors subdivided by stage $(n=15$ [I], 17 [II], 20 [III/IV]). \#\# $P=0.00022$ versus stage I and stage II, 2-way ANOVA. (H) Kaplan-Meier analysis of ERMS patients subdivided by ILK immunohistochemical score for relapsefree survival ( $n=17$ per group). $P=0.2643$ between groups, log-rank test. (I) Kaplan-Meier analysis of ERMS patients subdivided by ILK immunohistochemical score for overall survival ( $n=17$ per group). $P=0.0211$ between groups, log-rank test. 
A

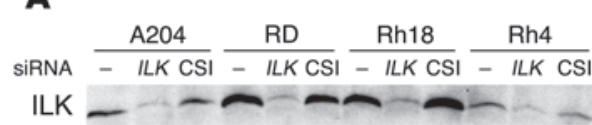

Vinculin

$\mathrm{K}-\mathrm{-m}-\mathrm{m}-\mathrm{m}$
B
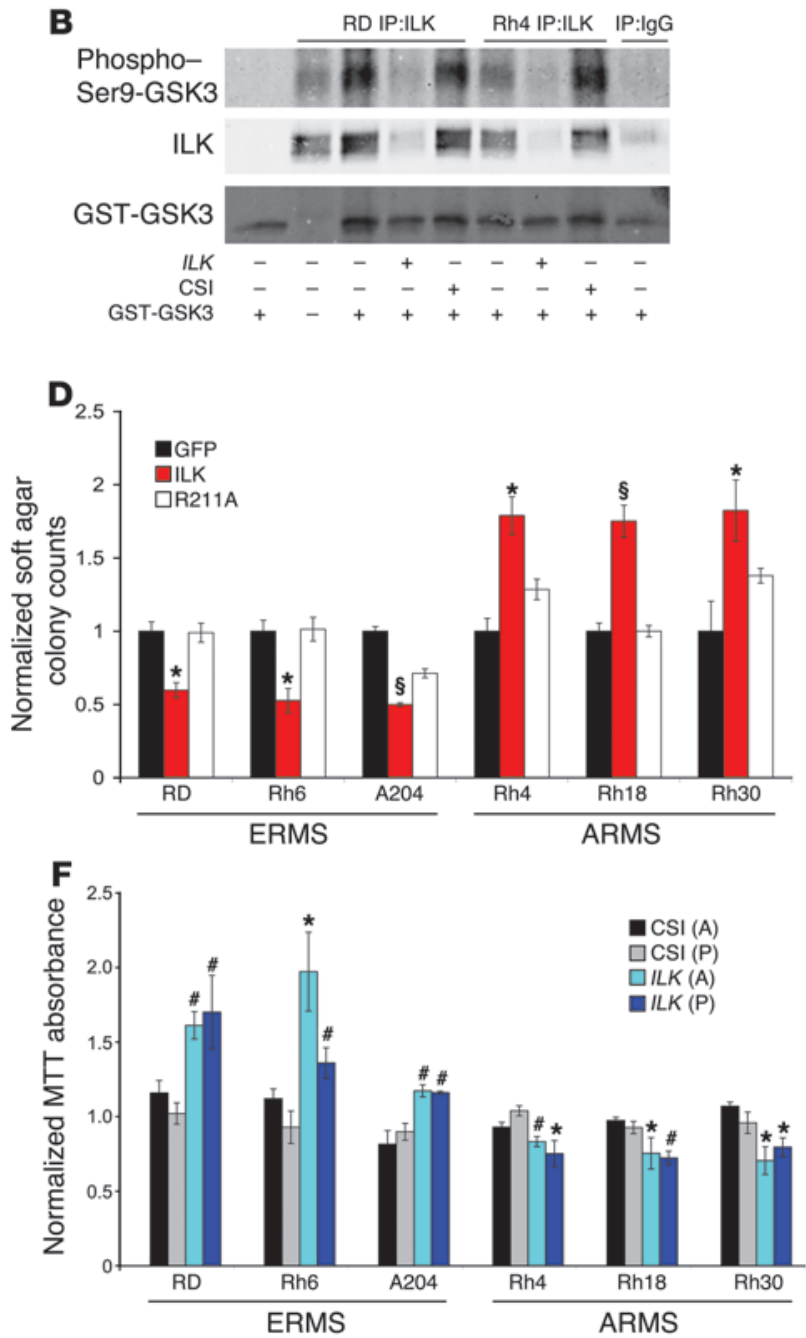

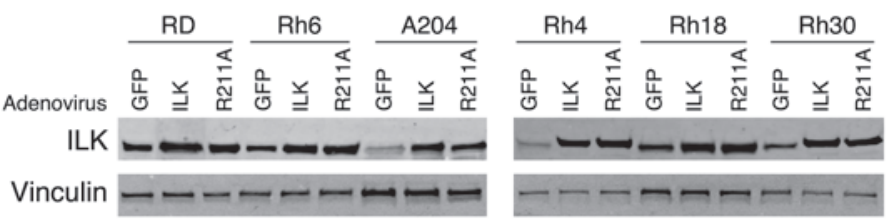

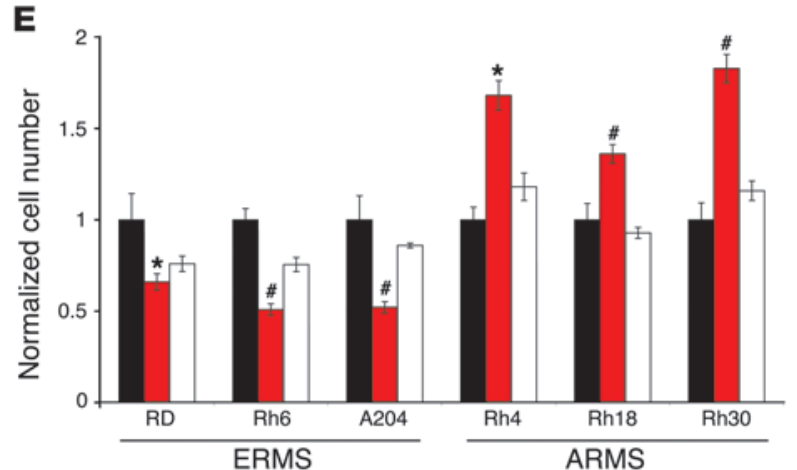

G

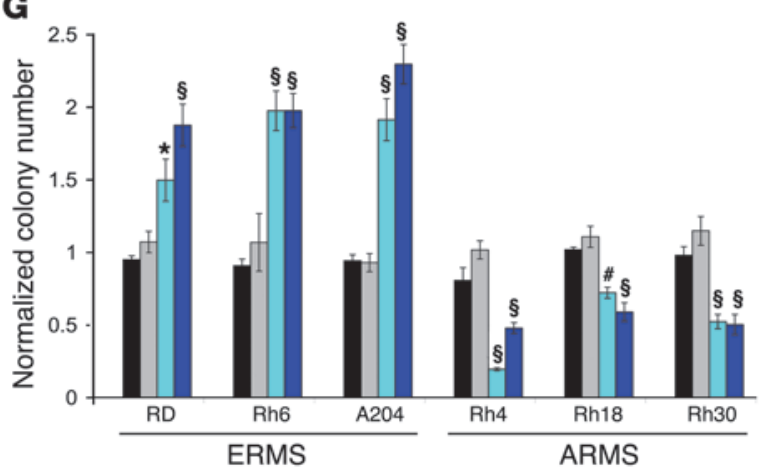

Figure 2

ILK promotes growth in ARMS and suppresses growth in ERMS. (A) Levels of ILK protein were assessed in RMS cells exposed to transfection reagent $(-)$, ILK, or nontargeted control siRNA (CSI) or with adenoviruses encoding GFP alone, ILK, or the ILK mutant R211A. Adenoviral treatments were made at $500 \mathrm{IU}$ for all cell lines except Rh4 (1,000 IU). Data are representative of 5 independent blots. (B and C) Comparative ILK in vitro kinase assays in RD ERMS and Rh4 ARMS cells exposed to ILK or control siRNAs (B) or to adenoviruses encoding GFP, ILK, or R211A (C). Data are representative of 4 independent blots. Lanes were run on the same gel but were noncontiguous (white line). (D and E) RMS cells were treated with adenoviruses encoding GFP, ILK, or R211A, and soft agar colony formation (D) and proliferation (E) were assessed $(n=4)$. ${ }^{*} P<0.05, \# P<0.01, \S P<0.001$ versus GFP and R211A, ANOVA. ( $\mathbf{F}$ and $\left.\mathbf{G}\right)$ RMS cells were treated with single siRNA duplexes (A) or with pools of $I L K$ or nontargeted control siRNA duplexes $(P)$, and proliferation $(\mathbf{F})$ and colony formation $(\mathbf{G})$ were assessed $(n=4)$. ${ }^{\star} P<0.05$, $\# P<0.01$, $\S P<0.001$ versus respective control siRNA, 2-way ANOVA.

in suppressed xenograft growth, with dramatic reductions in local invasion (Figure 3, B and D, and Table 1). Western blotting demonstrated reduced ILK levels at early (Supplemental Figure 3), but not late, time points (Figure 3E). Immunohistochemical analysis at sacrifice demonstrated that ILK siRNA-treated ERMS xenografts displayed dramatically elevated proliferation, as measured by Ki67, and moderately reduced apoptosis, as measured by
TUNEL, compared with controls, while ILK siRNA-treated ARMS xenografts displayed moderately reduced Ki67 staining and markedly enhanced TUNEL positivity (Figure 3E). Levels of cleaved caspase 3 in ILK siRNA-treated xenograft protein lysates confirmed the apoptotic effect in ARMS (Supplemental Figure 4). Visual examination of the tumor xenografts demonstrated increased vascularity in the RD ERMS ILK siRNA tumors, and reduced 
A
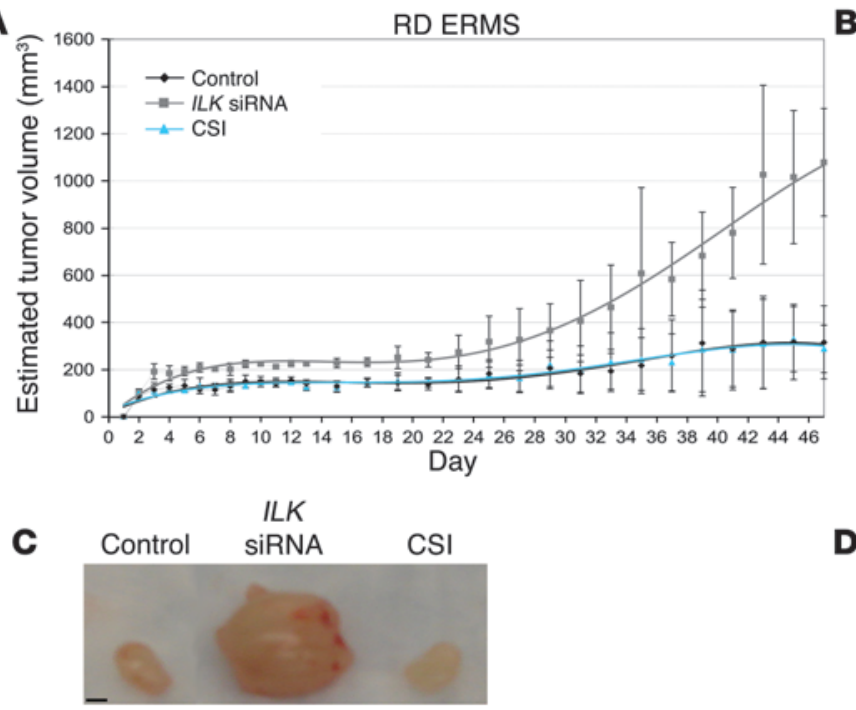

E

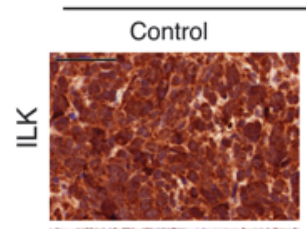

RD ERMS

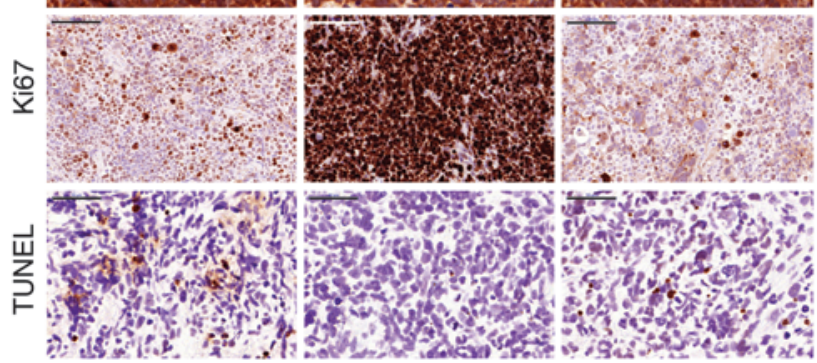

B

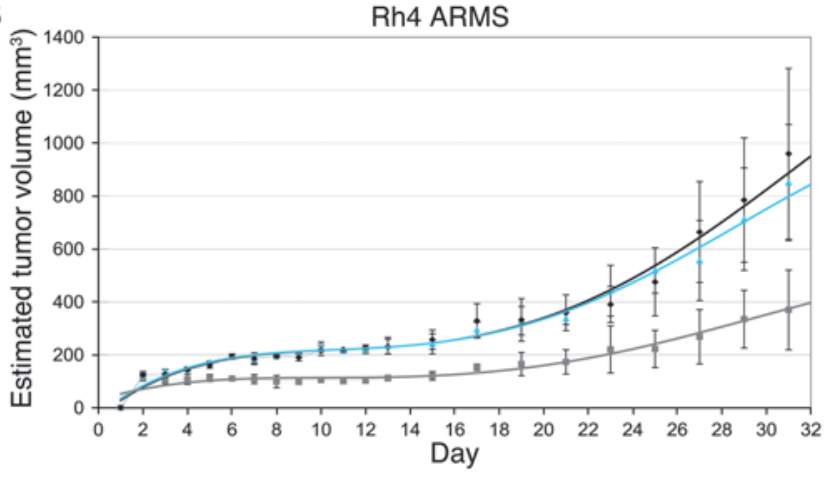

D

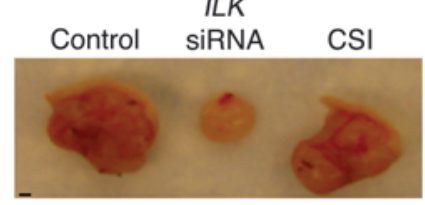

Rh4 ARMS

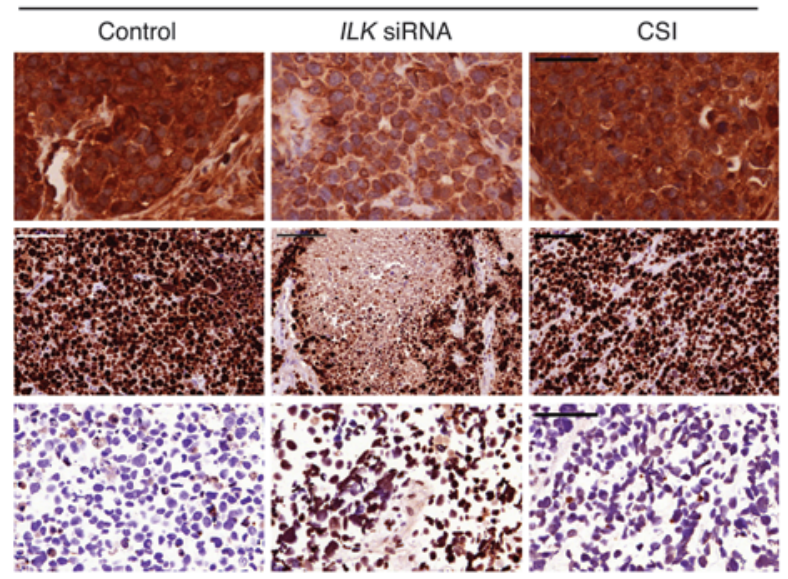

Figure 3

ILK has opposing functions in vivo. (A and B) Growth curves of RD ERMS (A) and Rh4 ARMS (B) xenografts. Cells were treated with transfection reagent (control) or with single ILK or control siRNA duplexes in vitro and implanted 72 hours later. Error bars represent SD. $n=6$ animals per cell line, per treatment group. $P<0.05$, ILK siRNA versus both controls at all points after day 2, 2-way ANOVA. (C and D) Representative wholetumor images of RD (C) and Rh4 (D) xenografts at sacrifice. Scale bars: $1 \mathrm{~mm}$. (E) Tumors were fixed and sectioned for immunohistochemical staining for ILK, Ki67, and TUNEL. Images are representative of 6 independent tumors per treatment, per cell line. Scale bars: 50 um (ILK, TUNEL); $100 \mu \mathrm{m}$ (Ki67).

vascularity in the Rh4 ARMS ILK siRNA tumors, which suggests that ILK modulation may affect angiogenesis (Figure 3, C and D). VEGF and Factor VIII staining demonstrated global and opposing effects on angiogenesis, with elevated VEGF and vascular staining in ILK siRNA-treated ERMS xenografts and reduced levels in ILK siRNA-treated ARMS xenografts relative to controls (Supplemental Figure 5). Taken together, these results suggest that ILK has opposite effects on ERMS and ARMS proliferation, apoptosis, and angiogenesis in vivo.

ILK function is sensitive to expression of PAX3-FKHR. The PAX3-FKHR fusion gene is characteristic of, and a negative prognostic factor in the diagnosis of, ARMS tumors (2). Furthermore, the expression of PAX-FKHR fusions in ERMS cells is sufficient to alter the transcriptional signature of ERMS cells to cluster with ARMS cells and tumors (3). Since the presence of a PAX-FKHR fusion gene is a critical difference between ERMS and ARMS, and ILK functions differently in ERMS and ARMS, we hypothesized that a PAX-FKHR fusion may alter the ERMS tumor-suppressive function of ILK. To test this hypothesis, we generated RD ERMS clones that stably express different levels of PAX3-FKHR (Figure 4A). $P A X 3-F K H R$-expressing clones demonstrated higher basal proliferation, clonogenicity, and soft agar colony formation than did vector controls (Supplemental Figure 6). ILK siRNA treatment of vector control RD ERMS cells demonstrated enhanced proliferation, clonogenicity, and soft agar colony formation, similar to RD parental cells, while similar treatment in PAX3-FKHR-expressing clones demonstrated diminished proliferation, clonogenicity, and soft agar colony formation (Figure 4, B-D). Moreover, overexpression of ILK reduced the proliferation, clonogenicity, and soft agar colony formation of vector controls, but induced these activities 


\section{Table 1}

ILK induces opposing effects on tumor invasion and metastasis in vivo

\begin{tabular}{lccc} 
Cell line & Treatment & Local invasion & Distant metastases \\
RD & Control & $0 / 6$ & $0 / 6$ \\
RD & ILK siRNA & $6 / 6$ & $3 / 6$ \\
RD & Control siRNA & $0 / 6$ & $0 / 6$ \\
Rh4 & Control & $4 / 6$ & $1 / 6^{A}$ \\
Rh4 & ILK siRNA & $0 / 6$ & $0 / 6$ \\
Rh4 & Control siRNA & $4 / 6$ & $0 / 6$ \\
\hline
\end{tabular}

Growth characteristics of RMS tumor xenografts treated with nothing (control) or with ILK or control siRNA were quantified at sacrifice. Local invasion denotes invasion into chest wall musculature. ${ }^{A}$ Outlier that was inadvertently placed intraperitoneally.

in PAX3-FKHR-expressing ERMS cells (Supplemental Figure 7). Similarly, transient transfection of PAX3-FKHR into primary MP59 mesenchymal progenitors effectively converted the growth-suppressive effect of ILK into a growth-promoting effect (Supplemental Figure 8). These results suggest that ILK function is sensitive to the genetic background of ERMS and ARMS cells.

ILK induces opposing regulation of the JNK/c-Jun signaling axis. Because the oncogenic or tumor-suppressive functionality of ILK was dependent on its kinase activity, we hypothesized that ILK regulates these opposing effects via dysregulation of kinase targets. Analysis of canonical ILK-regulated phosphorylation sites, such as Ser473-Akt and Ser9-GSK3 $\beta(32,33)$, demonstrated similar reductions in both ARMS and ERMS cells in response to ILK siRNA (data not shown), which suggests that alternative pathways were involved in the disparate growth effects in ERMS versus ARMS. ILK has previously been demonstrated to indirectly regulate the phosphorylation of members of the MAPK signaling family $(14,34,35)$. Analysis of the phosphorylated MAPKs ERK1 and ERK2 demonstrated similar results to Akt and GSK3 $\beta$ (data not shown).

In contrast, phosphorylation of the JNK proteins and their target, c-Jun, were elevated in ILK siRNA-treated ERMS cells and suppressed in ILK siRNA-treated ARMS cells (Figure 5A). Levels of p46 and p54 JNK proteins were dissimilar between the different RMS cell lines (Figure 5A; compare RD and Rh4 with Rh6 and Rh18), suggesting that differences in mRNA and protein expression and stability patterns from the $3 \mathrm{JNK}$ genes are found in these cells. No differences in another JNK target, pThr71-ATF2, were observed in ERMS or ARMS cells in response to similar ILK depletion (data not shown). To assess whether these proteins were also activated in vivo, we measured the levels of phosphorylated c-Jun and JNK in xenografts and demonstrated induction of both phosphoc-Jun and phospho-JNK in ILK siRNA-treated ERMS xenografts and suppression in ILK siRNA-treated ARMS xenografts (Figure $5 B$ ). Similar to our in vitro results, no effects were detected on the canonical ILK targets Ser473-Akt and Ser9-GSK3 $\beta$ in vivo (data not shown). To measure c-Jun/activator protein 1 (c-Jun/AP-1) activity, we analyzed 7 transcriptional targets by quantitative RT$\mathrm{PCR}$, including genes both transactivated (SDF1 $\alpha, C y c A 2$, and $C y c D 1)$ and repressed (FasL, $p 21^{C I P 1}, p 16^{I N K 4 A}$, and $\left.p 14^{A R F}\right)$ by active c-Jun/AP-1 complexes. We found that c-Jun/AP-1 activity was induced in response to ILK siRNA in ERMS cells and suppressed in ARMS cells (Figure 5C). These observations, in cooperation with our data indicating that $P A X 3-F K H R$ expression could con- vert ILK from a tumor suppressor in ERMS cells to an oncogene in ARMS cells, led us to examine the phosphorylation status of JNK and c-Jun in PAX3-FKHR-expressing RD clones. Similar to RD parental cells, RD vector controls treated with ILK siRNA displayed enhanced phosphorylation of JNK and c-Jun (Figure 5D). In contrast to the vector controls, and similarly to ARMS cells, PAX3-FKHR-expressing RD cells displayed reduced levels of phospho-JNK and phospho-c-Jun when treated with ILK siRNA (Figure $5 \mathrm{D})$. These data indicate that $I L K$ siRNA induces phosphorylation of JNK and c-Jun in ERMS and represses in ARMS cells in vitro and in murine models. Furthermore, these data demonstrate that PAX3-FKHR can convert the effect of ILK on regulation of JNK and c-Jun phosphorylation. To investigate whether this interaction between ILK and JNK was direct or indirect, we performed coimmunoprecipitation analysis of ILK, with Western blotting for JNK (data not shown). These experiments failed to demonstrate the presence of JNK in ILK immunoprecipitates, which indicates that the interaction of ILK and JNK may be indirect in nature.

To establish whether similar alterations in the JNK/c-Jun signaling axis could be found in vivo in human RMS tumors, we examined the levels of phosphorylated JNK by immunohistochemistry. Immunostaining revealed no difference in the level of phospho-JNK between primary ARMS and ERMS tumors (data not shown). However, separation of ARMS tumors into groups with low and high ILK expression demonstrated that JNK phosphorylation increased in tumors expressing high levels of ILK (Figure 5E). In contrast, separation of ERMS tumors into similar groupings demonstrated that JNK phosphorylation was reduced in tumors expressing high levels of ILK (Figure 5F). Finally, because ILK levels were reduced in stage III/IV ERMS tumors (Figure 1G), we examined phospho-JNK levels in ERMS, subdivided by stage. Phospho-JNK levels increased in stage III/IV primary ERMS tumors compared with stages I and II (Figure 5G). These data demonstrated similarities among the in vitro, murine, and primary tumor models and that phosphorylated JNK levels correlated positively and negatively with ILK level in primary human ARMS and ERMS tumors, respectively.

$J N K / c$-Jun pathway mediates the tumor-suppressive effect of ILK. To assess whether the induction of JNK and c-Jun phosphorylation was causally related to the increased proliferation of ERMS cells in response to loss of ILK, we evaluated the effects of coinhibition of JNK and ILK. We found that the induction of proliferation induced by ILK siRNA was dramatically reduced by treatment with the JNK inhibitor SP600125, but not the inactive JNK inhibitor $\mathrm{N}^{1}$-Methyl1,9-pyrazoloanthrone or an equal volume of vehicle, in 3 ERMS cell lines (Figure 6A). Western blotting confirmed that SP600125 reduced c-Jun phosphorylation on the Ser63 JNK target site in Rh6 and RD ERMS cells (Figure 6B and Supplemental Figure 9). To elucidate the specific JNK mediating this effect, we used siRNA pools to transiently deplete JNK1 and JNK2 alone or together with ILK in ERMS cells. Loss of JNK1, but not JNK2, suppressed ILK siRNA-stimulated proliferation, and triple knockdown of JNK1, JNK2, and ILK had no increased effect relative to ILK and JNK1 knockdown alone (Figure 6C). Western blotting confirmed that knockdown of JNK1, but not JNK2, led to suppression of both endogenous and ILK siRNA-stimulated c-Jun phosphorylation in Rh6 and RD ERMS cells (Figure 6D and Supplemental Figure 10). Experiments in RD vector controls similarly demonstrated that knockdown of JNK1, but not JNK2, led to suppression of ILK siRNA-stimulated proliferation and c-Jun phosphorylation (Supplemental Figure 11). Intriguingly, JNK1 siRNA did not further 
A
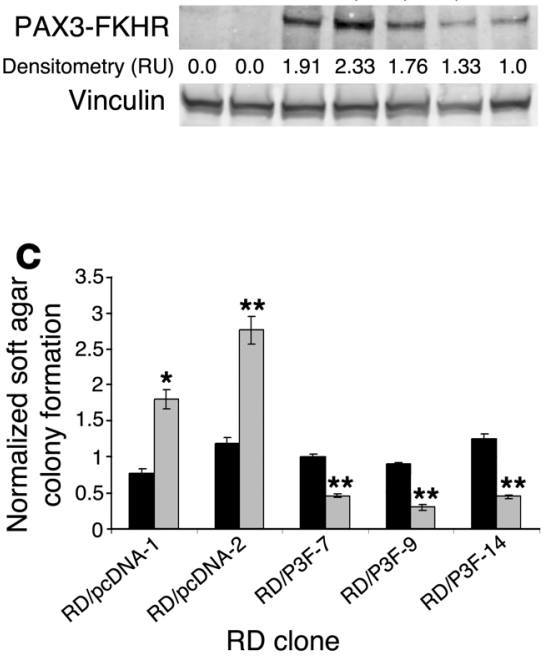

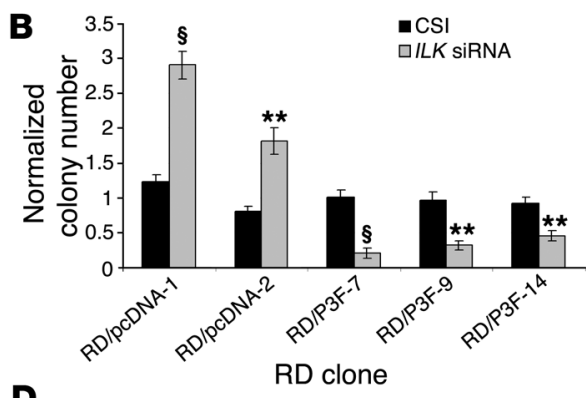

D

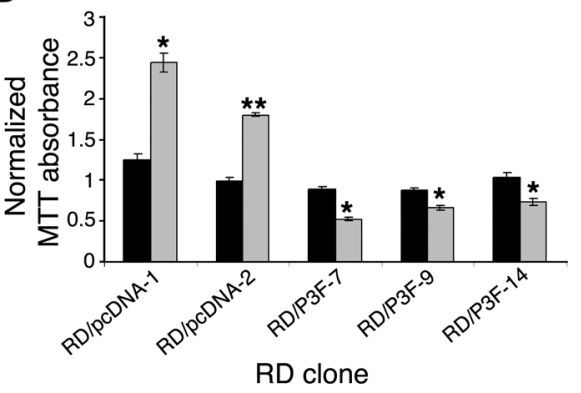

Figure 4

PAX3-FKHR expression converts ERMS cells to exhibit ARMS-like responses to ILK depletion. (A) PAX3-FKHR protein expression levels in vector (RD/pcDNA-1, RD/ pcDNA-2) and PAX3-FKHR-expressing (RD/P3F-7, RD/P3F-9, RD/P3F-14) clones were determined by Western blotting. Rh4 and Rh30 ARMS cells are shown as positive controls for PAX3-FKHR expression. Data are representative of 4 independent lysates. Densitometry values show the average of 4 independent blots and are normalized to Rh30 ARMS cells. (B-D) RD clones were transfected with nothing (control; not shown) or with ILK or control siRNAs, and colony (B), soft agar (C), and MTT (D) assays were performed. ${ }^{*} P<0.05,{ }^{* *} P<0.01,{ }^{\S} P<0.001$ versus untransfected and control siRNA groups, 2-way ANOVA. reduce the $I L K$ siRNA-stimulated suppression of proliferation and c-Jun phosphorylation in RD PAX3-FKHR clones (Supplemental Figure 11). These observations suggested that JNK1 is required for the phosphorylation of c-Jun and the proliferation effect downstream of ILK depletion in ERMS cells (Figure 6, C and D, and Supplemental Figures 10 and 11). Moreover, interruption of c-Jun/AP-1 activity using an adenovirally expressed dominant-negative c-Jun transactivation domain mutant (TAM67) suppressed ILK siRNA-induced proliferation, clonogenicity, and soft agar colony formation (Figure 6E and Supplemental Figure 12) in all 3 ERMS cell lines. Intriguingly, Western blotting of lysates demonstrated the presence of cleaved caspase 3 in TAM67-expressing, ILK siRNA-treated Rh6 cells (Figure 6F), which indicates that loss of ILK and c-Jun/AP-1 signaling results in enhanced apoptosis in this cell line. These results support our finding that the induction of JNK/c-Jun signaling was involved in the increased growth observed upon ILK depletion in ERMS cell lines.

JNK1 overexpression restores the ERMS phenotype to ARMS cells. Our data suggested that expression of JNK1 was critical to the activation of c-Jun in ERMS cells treated with ILK siRNA. To further explore the role of JNK1, we assessed expression levels of the JNK isoforms with kinase specificity for c-Jun (36) in a panel of RMS cell lines. We observed moderately elevated expression of $J N K 1 B 1$ and JNK1B2 transcripts in ERMS cells relative to ARMS cells (Figure 7A). Furthermore, expression of the JNK1B isoforms were reduced in RD PAX3-FKHR clones (Figure 7B). These data suggested that JNK1 $\beta$ isoforms are under $P A X 3-F K H R$ control and that their reduced expression is characteristic of ARMS cells. These findings led to the hypothesis that restoring expression of JNK1 in ARMS cells may result in an opposing phenotypic switch in ILK activity from that of an oncogene to a tumor suppressor. To address this hypothesis, we overexpressed JNK1 in Rh18 and Rh30 ARMS cells in the setting of ILK siRNA. Clonogenicity and proliferation of ARMS cells treated with ILK siRNA was reduced, but could be rescued in the setting of JNK1 overexpression (Figure 7C and Supplemental Figure 13). Furthermore, these alterations in proliferation and clonogenicity were associated with selective induction of c-Jun phosphorylation (Figure 7D) not seen in JNK1overexpressing cells alone. In total, these experiments provide support to the hypothesis that PAX3-FKHR is capable of reversing the function of ILK from a tumor suppressor to an oncogene in ERMS and mesenchymal progenitor cells through the downregulation of JNK1. This finding was further supported by our demonstration that increased expression of JNK1 in ARMS cells caused the reversal of ILK activity from an oncogenic to a tumor-suppressive function through activation of JNK/c-Jun signaling.

\section{Discussion}

The mechanisms by which tumor-suppressive and oncogenic proteins are capable of inducing opposite effects are beginning to be elucidated. The acquisition of a mutation in some genes is sufficient to transform primary cells, such as is found with tumor protein, $53 \mathrm{kDa}$ (TP53; ref. 37). In other cases, alterations in domain structure can confer aberrant activities, as with the tumor suppressor $\mathrm{p} 27^{\mathrm{kip} 1}$, which has oncogenic activity when the cdkbinding domain is removed experimentally (38). Further, genetic context can determine the function of a protein; for example, the multiple endocrine neoplasia 1 (MEN1) gene is a tissue-specific oncogene or tumor suppressor (39), and in glioblastoma, STAT3 can function as an oncogene in the setting of mutant EGFR or as a tumor suppressor in the setting of mutant phosphatase and tensin homolog deleted on chromosome 10 (PTEN; ref. 40). RMS is subdivided into 2 primary and distinct histologies, ERMS and ARMS. While these tumor subtypes are suspected to arise as a result of genetic damage to a common mesenchymal or committed myogenic precursor, the 2 histologies display a large number of clinical, genetic, and molecular differences, including the expression of PAX-FKHR chromosomal fusions in ARMS. These differences suggest that the histologies represent distinct, but linked, genetic backgrounds, a notion supported by previous cDNA microarray studies (3). We demonstrated here that ILK function as an oncogene or tumor suppressor was dependent on cell context, which was reflected by roles distinguished by tumor histology and molecular characteristics. 

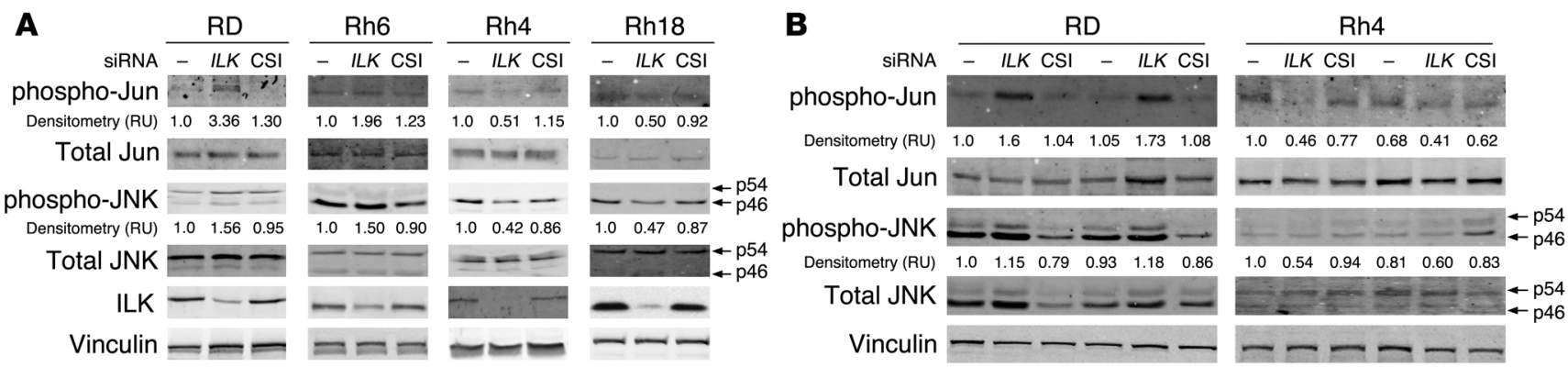

C
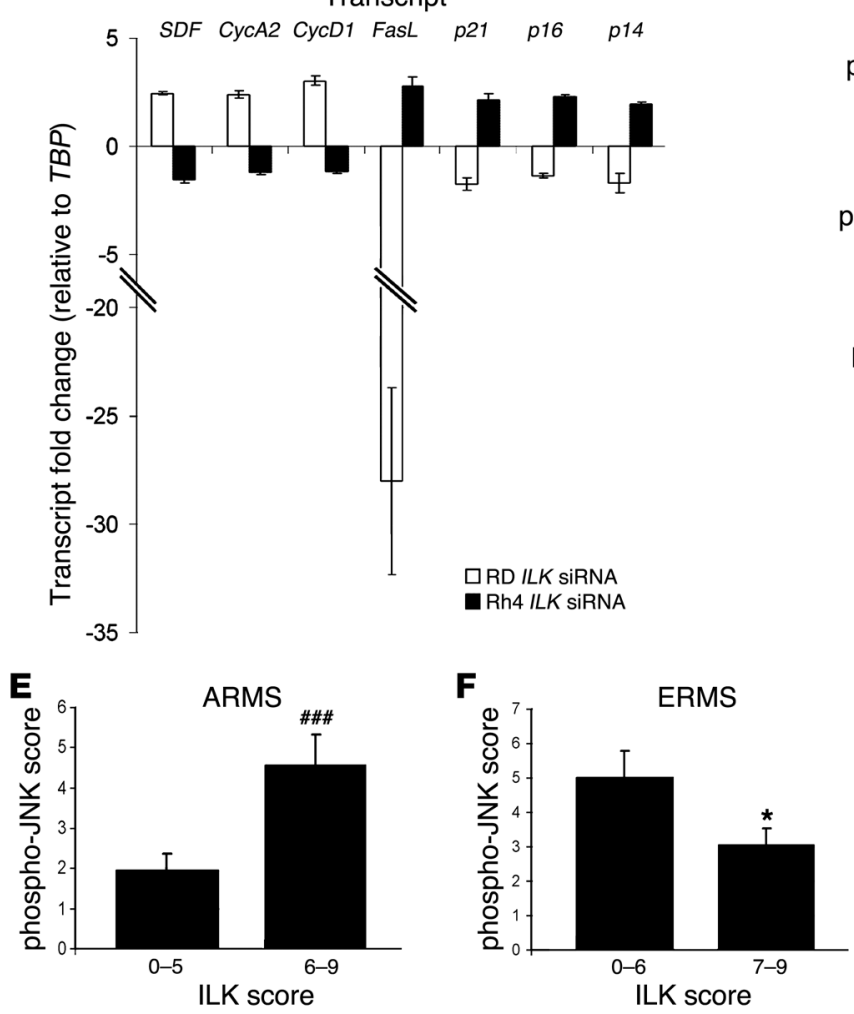

D
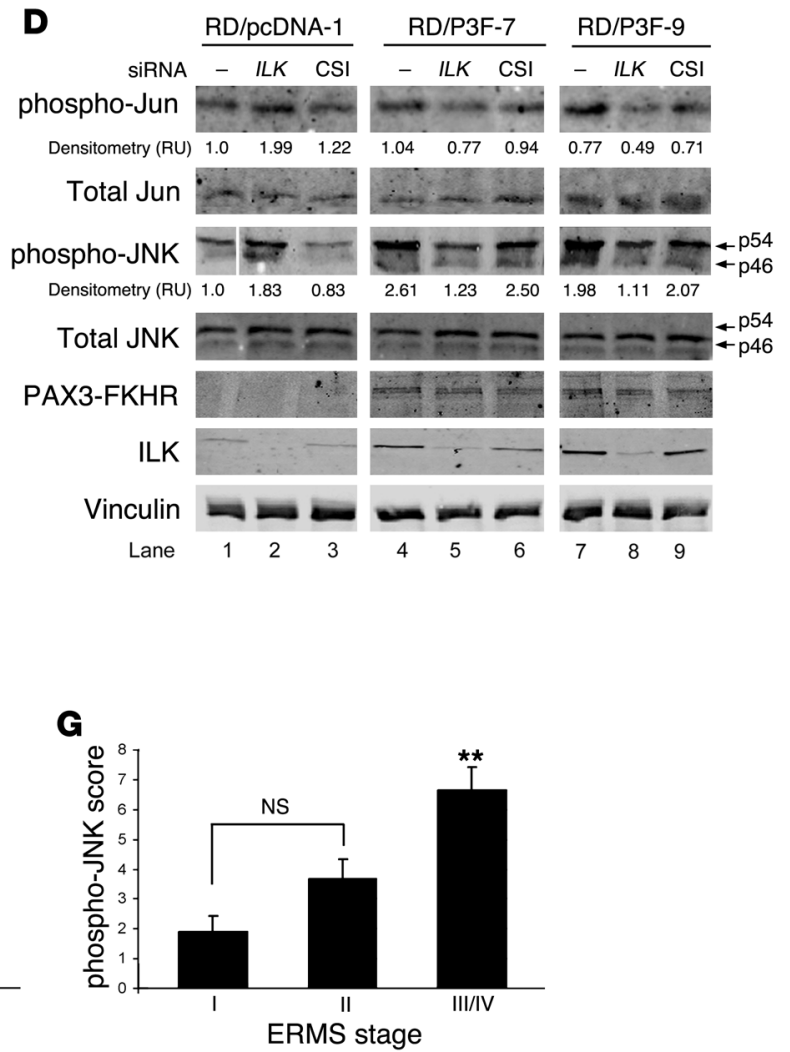

Figure 5

Loss of ILK induces opposing effects on the JNK/C-Jun signaling axis sensitive to PAX3-FKHR in vitro and in vivo. (A) RD and Rh6 (ERMS) as well as Rh4 and Rh18 (ARMS) cell lines were transfected with nothing or with ILK or control siRNAs and lysed for Western blotting. Arrows show p54 and p46 JNK proteins. Blots and densitometry represent the average of 4 independent experiments. (B) RMS xenografts were lysed for Western blotting. Data are representative of 6 tumors per treatment, per cell line. Arrows show p54 and p46 JNK proteins. (C) RD (ERMS) and Rh4 (ARMS) cells were transfected with ILK or control siRNAs and lysed for RNA. Quantitative RT-PCR for c-Jun/AP-1-regulated transcripts was performed $(n=4)$. Values denote transcript fold change relative to control siRNA treatment. All differences were significant $(P<0.001)$ compared with internal control siRNA. (D) RD vector and PAX3-FKHR clones were transfected with nothing or with ILK or control siRNAs. Lysates were prepared for Western blotting. Data are representative of 3 independent blots and normalized against lipofectamine-treated RD/pcDNA-1 cells. Arrows denote p46 and p54 JNK proteins. Phospho-JNK lanes were run on the same gel but were noncontiguous (white line). (E) ARMS primary tumors were divided into low $(0-5 ; n=22)$ and high $(6-9 ; n=13)$ ILK score groups, and phospho-JNK score was determined. \#\#\# $P=0.0031$ versus low ILK score. (F) ERMS primary tumors were divided into low $(0-6 ; n=17)$ and high $(7-9 ; n=19)$ ILK score groups, and phospho-JNK score was determined. ${ }^{*} P=0.038$ versus low ILK score. (G) ERMS samples were divided by tumor stage, and phospho-JNK score was determined $(n=10[\mathrm{I}] ; 12[\mathrm{II}] ; 11[\mathrm{III} / \mathrm{IV}]) .{ }^{*} P<0.01$ versus stage I and stage II, ANOVA.

Here, we demonstrated tumor-suppressive and oncogenic functions of ILK in ERMS and ARMS, respectively, and showed that the difference in outcome depended on what we believe to be a previously unknown interaction between ILK and JNK1 pathway signaling. The major findings in this study are as follows. First, ILK functioned as a tumor suppressor in ERMS cells and as an oncogene in ARMS cells in vitro and in vivo. Second, loss of ILK in ERMS induced signaling through a JNK/c-Jun cascade, suppression of which resulted in dramatic decreases of growth. Third, loss of ILK in ARMS led to suppressed JNK/c-Jun signaling and growth. Fourth, introduction of the ARMS fusion gene PAX3-FKHR into ERMS cells converted ILK into an oncogene and suppressed JNK/ 
A

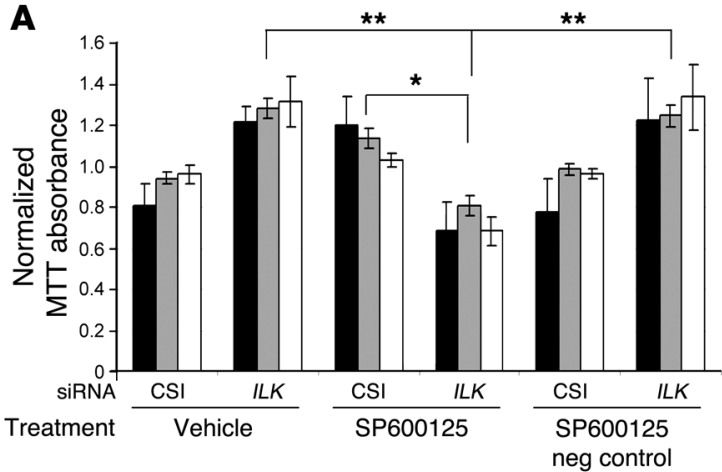

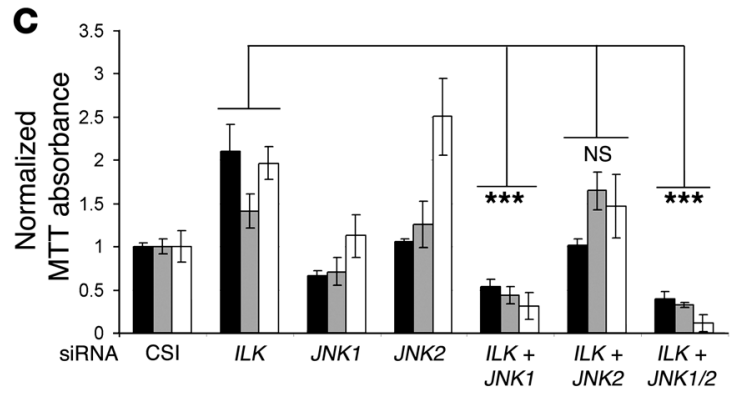

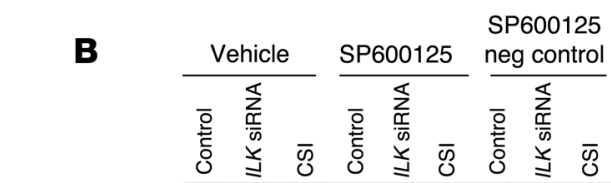

phospho-Jun

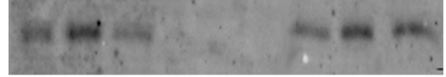

$\begin{array}{llllllllll}\text { Densitometry (RU) } & 1.0 & 1.30 & 0.85 & 0.20 & 0.28 & 0.16 & 1.07 & 1.38 & 1.04\end{array}$

Total Jun
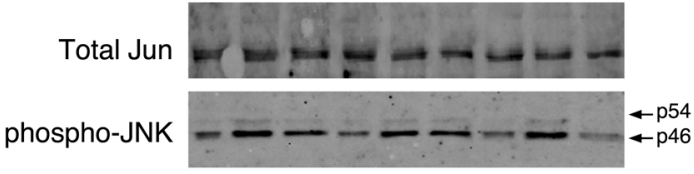

$\begin{array}{llllllllll}\text { Densitometry (RU) } & 1.0 & 1.60 & 1.12 & 0.93 & 1.41 & 1.14 & 1.10 & 1.65 & 0.93\end{array}$

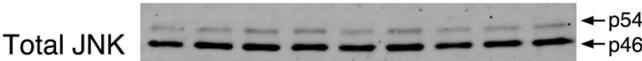

ILK

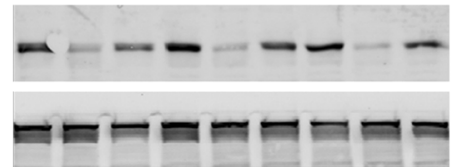

Vinculin ーேーーーーーーーー

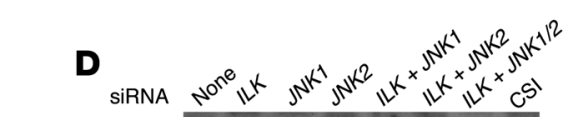

phospho-Jun

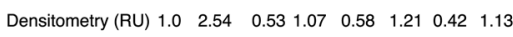

Total Jun

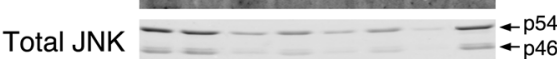

ILK

Vinculin
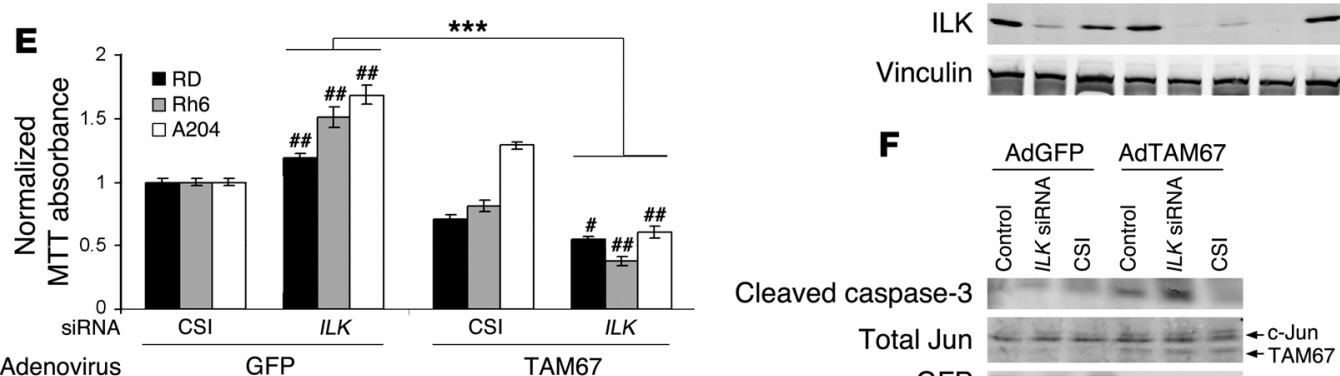

Cleaved caspase- 3

$\mathbf{F}$

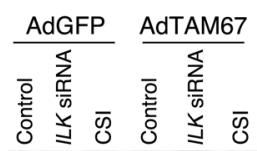

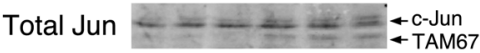

GFP

ILK

Vinculin $=--=$

\section{Figure 6}

The JNK pathway is a transducer of the ERMS response to ILK depletion. (A) ERMS cell lines were transfected with nothing (control; not shown) or ILK or control siRNAs and exposed to $20 \mu \mathrm{M}$ SP600125, inactive JNK inhibitor N1-Methyl-1,9-pyrazoloanthrone (SP600125 negative [neg] control), or equal concentrations of DMSO vehicle for 1 hour before MTT assay $(n=4)$. Data are normalized to lipofectamine-transfected vehicletreated controls. ${ }^{\star} P<0.05,{ }^{\star *} P<0.01$, ANOVA. (B) Rh 6 cells were treated as in A, but lysed for Western blotting. Data are representative of 4 independent replicates. Arrows show p54 and p46 JNK proteins. (C) ERMS cells were transfected with ILK, JNK1, JNK2, or control siRNAs and analyzed by MTT assay 4 days after transfection $(n=4)$. Data are normalized to lipofectamine-transfected controls. ${ }^{* \star *} P<0.001$, ANOVA. (D) Rh6 cells were treated as in C, but lysed for Western blotting. Data are representative of 4 independent replicates. Arrows show p54 and p46 JNK proteins. (E) ERMS cells were transfected with ILK or control siRNAs and exposed to adenovirus encoding TAM67 or GFP (1,000 IU), and cells were analyzed by MTT assay 4 days after transfection $(n=4)$. Data are normalized to lipofectamine-transfected GFP adenovirus-treated

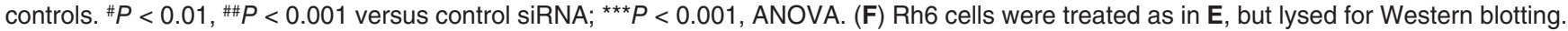
Data are representative of 4 independent replicates. Arrows show endogenous c-Jun and TAM67 proteins.

c-Jun signaling when ILK was depleted. Finally, restoration of JNK1 in ARMS cells reestablished ERMS-type tumor-suppressive signaling when ILK was depleted. While our findings suggest that the interaction between ILK and JNK1 is indirect, further work is required to establish the intermediates linking these 2 proteins. Collectively, these findings suggest that ILK functions distinctly as an oncogene or tumor suppressor in the context of key genetic alterations associated with ARMS (Figure 8).

Our data revealed that in ERMS tumors, loss of ILK potentiated signaling through a JNK/c-Jun signaling axis, resulting in cellular proliferation and survival independent of the canonical ILK signaling targets PKB/Akt and GSK3 $\beta$. Intriguingly, we found 

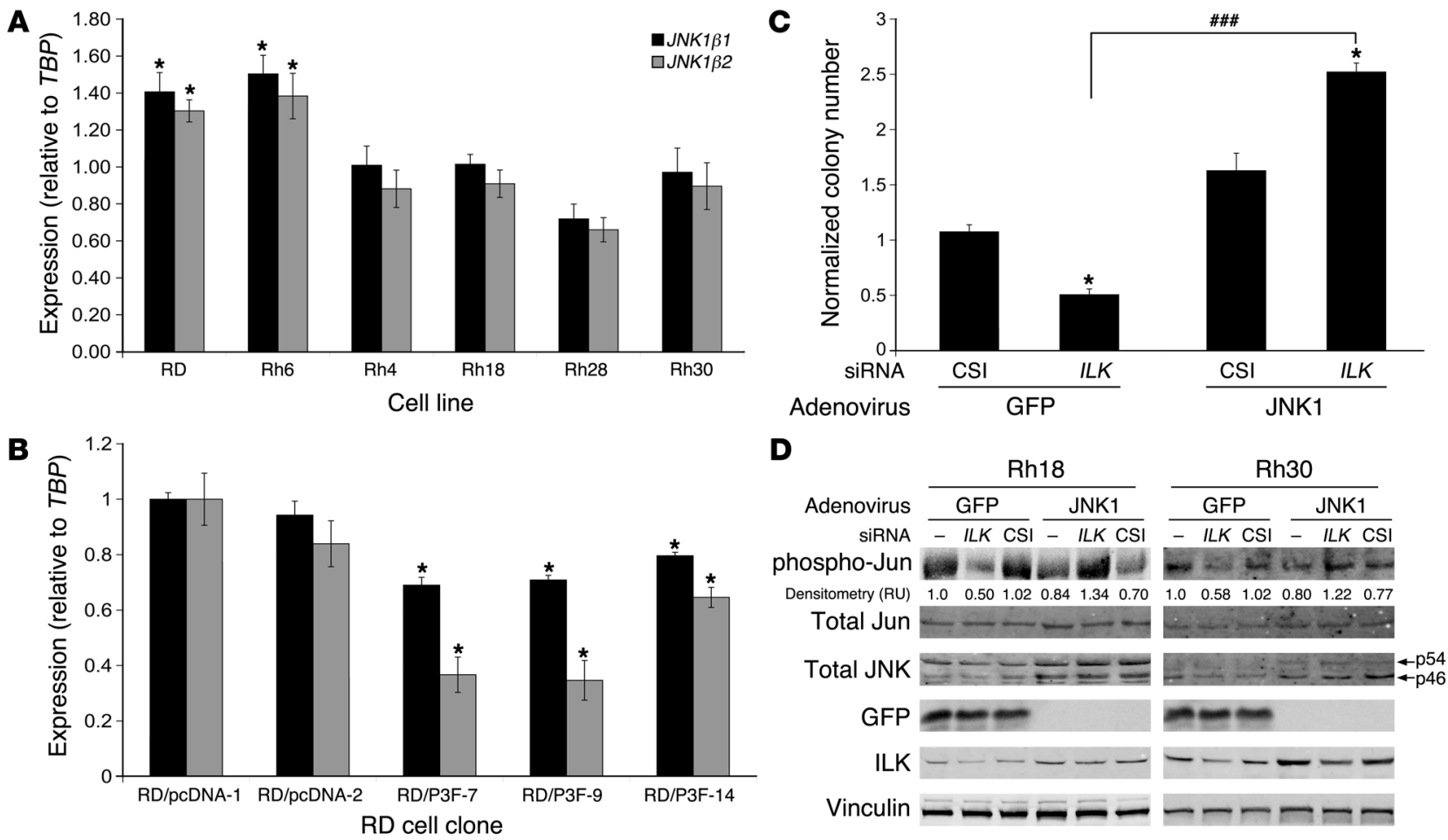

Figure 7

Restored expression of JNK1 reverses the ARMS response to ILK depletion. (A) Quantitative RT-PCR to JNK1B1 and JNK1B2 isoforms in RMS cells $(n=4)$. ${ }^{*} P<0.05$ versus ARMS cell control, ANOVA. (B) Quantitative RT-PCR to JNK1B1 and JNK1B2 isoforms in RD vector and $P A X 3-F K H R$ clones $(n=4) .{ }^{*} P<0.05$ versus vector control. (C) Rh30 ARMS cells were transfected with ILK or control siRNAs and after 48 hours were infected with $100 \mathrm{IU}$ adenovirus encoding JNK1 or GFP. Cells were analyzed by colony assay 14 days after transfection $(n=4)$. Data are normalized against lipofectamine-transfected, GFP adenovirus-infected controls. ${ }^{*} P<0.05$ versus respective control; \#\# $P<0.001$. (D) Rh18 and Rh30 cells were treated as in $\mathbf{C}$, but lysed for Western blotting. Cells were transfected with nothing or with ILK or control siRNAs. Data are representative of 4 independent replicates. Arrows show p46 and p54 JNK proteins.

(a) reduced mRNA and protein expression of ILK in high-stage human ERMS tumors (Figure 1, D and G); (b) increased metastases in ILK-depleted ERMS xenografts (Table 1); (c) activated JNK/ c-Jun signaling in ILK-depleted ERMS xenografts (Figure 5B); and (d) an inverse correlation between ILK and phosphorylated JNK levels in primary human ERMS (Figure 5, F and G). In the context of previous reports demonstrating a critical role for c-Jun activity in the maintenance of the ERMS tumorigenic phenotype $(21,24$, 41 ) as well as data suggesting that ILK may function as a tumor suppressor in vivo $(4,17)$, our present findings implicate ILK as a potential metastasis-suppressor protein in ERMS that functions by regulating JNK/c-Jun signaling and suggest that exploration of JNK1 or c-Jun inhibitors may be warranted in metastatic ERMS.

In contrast, loss of ILK blunted signaling through this axis in ARMS cells, leading to reductions of proliferation and increased tumor cell apoptosis. Our data therefore suggest that ILK may function as a proto-oncogenic kinase in ARMS cells, with reduced ILK levels in vitro and in vivo causing diminished tumor growth and angiogenesis and increased levels of tumor cell apoptosis. These data are supported by a recent study demonstrating that ILK kinase inhibition in ARMS cell lines reduces cell viability (42). In contrast to ERMS, we observed no changes in ILK expression in high-stage ARMS, which suggests that basal ILK function may be sufficient to drive oncogenic signaling in these tumors. Intriguingly, since ARMS and PAX3-FKHR-transfected ERMS cells dem- onstrated reduced endogenous levels of JNK1, ILK depletion in ERMS cells induced activation of JNK1 specifically, and restoration of JNK1 to ARMS cells was sufficient to convert ILK from an oncogene to a tumor suppressor, our data indicate that expression levels of JNK1 are critical to ILK function. Furthermore, since the ARMS-specific fusion PAX3-FKHR reduced JNK1 mRNA levels, we suggest that the genetic background of ARMS and ERMS is sufficient to induce disparate signaling and growth patterns in response to ILK depletion.

Expression of PAX3-FKHR in ERMS and mesenchymal progenitor cells was sufficient to convert the tumor-suppressive signaling of ILK into oncogenic signaling. Retroviral expression of PAX3-FKHR in ERMS cells induced transcriptional changes, resulting in an ARMS-like expression signature, including the upregulation of the JNK pathway genes ASK1, HPK, and c-Jun (3). Coincident with the loss of a tumor-suppressive and the acquisition of an oncogenic role for ILK in these clones were elevated ILK levels (Figure 5D), reduced JNK1B1/2 expression (Figure 7B), and higher basal phosphorylation of JNK proteins, likely representing JNK2 and JNK3 gene products (Figure 5D). These clones failed to phosphorylate c-Jun on Ser63 in response to ILK depletion, consistent with prior reports that JNK1 $\beta 1$ and JNK1 $\beta 2$ demonstrate substrate specificity toward c-Jun (36). Furthermore, disrupting JNK after depleting ILK in ERMS cells led to reductions in proliferation, and disruption of c-Jun activity induced apoptosis (Figure 6 and Supplemental Figure 11). 


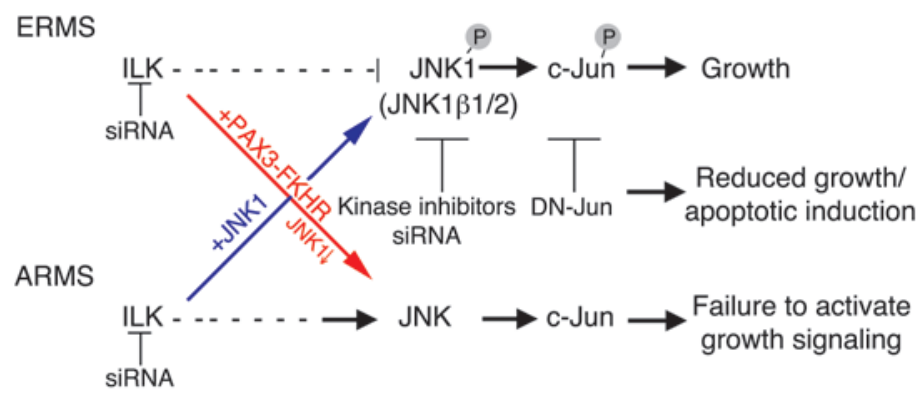

Figure 8

ILK oncogenic and tumor-suppressive signaling in RMS. In ERMS, ILK endogenously suppresses phosphorylation and activation of JNK1. Reductions of ILK result in JNK1 activation, which in turn activates c-Jun, leading to an increase in cell growth. Interruption of this induced JNK/C-Jun signaling then causes growth reductions or induction of apoptosis. In ARMS, ILK induces JNK phosphorylation, resulting in activation of c-Jun and growth. Furthermore, PAX3-FKHR downregulates baseline expression of JNK1 in ARMS, which causes failure to activate JNK/c-Jun signaling when ILK is depleted, resulting in reduced proliferation and apoptosis. Restoration of JNK1 expression in ARMS leads to reestablishment of this survival axis, and in the setting of ILK depletion, JNK1 signaling is activated, resulting in increased survival through activation of the c-Jun oncogene, as in embryonal cells. DN-Jun, dominant-negative c-Jun.

These findings echo previous studies $(22,41)$ and further indicate that activity of c-Jun is of critical importance in RMS cells.

It is interesting to note that transient ILK depletion in the RD and Rh4 cell lines resulted in a sustained in vivo effect on tumor growth, apoptotic and angiogenic characteristics (Figure 3 and Supplemental Figures 3 and 4), and the JNK/c-Jun signaling pathway (Figure 5B), while ILK expression largely returned to baseline levels (Figure 3E). Whether this represents an effect on the early establishment of the xenograft, or a more permanent alteration by siRNA in the signaling properties of these cells in vivo, remains a focus of future inquiry.

It is likely that our finding of a tumor-suppressive role for ILK extends beyond ERMS. Previous studies have suggested that a region of LOH lies over the ILK locus in numerous cell types, including ERMS, breast carcinoma, and non-small cell lung carcinoma (4). Similarly, ILK functions as a tumor suppressor in MDAMB-435 breast carcinoma cells, which display LOH at this locus (17). We have extended these results to suggest that ILK functions as a growth suppressor in one putative progenitor cell population for RMS tumors. Intriguingly, expression of PAX3-FKHR in these cells rescued the dampened growth induced by ILK overexpression, similar to ERMS cells. These results are strongly indicative of a conserved role for ILK as a tumor suppressor in certain mesenchymal lineage cells and are consistent with similar data demonstrating ILK-induced differentiation of rat myoblasts (12) and cooperative interactions between ionizing radiation and ILK in the stimulation of apoptosis of HL60 acute promyelocytic leukemia and Jurkat $T$ cells (16).

In summary, our study provides insights into a dual function for ILK in RMS as both an oncogene and a tumor suppressor. The mechanism by which this occurs was found to involve ILK interactions with a JNK/c-Jun pathway, which were altered by the ARMSspecific chromosomal fusion gene PAX3-FKHR. PAX3-FKHR induced a transition in ILK signaling from tumor suppressor to oncogene by downregulating JNK1 expression and suppressing signaling downstream of JNK1 to c-Jun. Furthermore, the restoration of JNK1 into ARMS cells completed the opposite switch in ILK function: from an oncogene to a tumor suppressor. In providing mechanistic insight into the role of ILK in RMS, as well as other tumors, our findings point to further complexities in the utility of ILK inhibition in tumors of these types and suggest that patient stratification on the basis of ILK and JNK1 expression signatures may be required to determine which patients may benefit from ILK inhibition as a form of targeted antitumor therapy.

\section{Methods}

Tumor samples and cell lines. Paraffin-embedded and snap-frozen RMS primary tumors were obtained from the archives of the Department of Pathology of the Hospital for Sick Children. Further RMS tissue microarrays were obtained from the Comprehensive Human Tissue Network. Patients consented to the use of their tissues in research projects, and this project received institutional ethics approval from the Hospital for Sick Children research ethics board (no. 1000004963). RD and A204 cells were purchased from the American Tissue Culture Collection. Rh4, Rh18, Rh28, and Rh30 cells were a gift of T. Look (Dana-Farber Cancer Institute, Boston, Massachusetts, USA). All cells were cultured in DMEM with 10\% FBS (Invitrogen) and confirmed to be free of Mycoplasma. Details of other cells are provided in Supplemental Methods.

Reagents. Adenoviruses encoding ILK and GFP were previously described (43). Modifications to yield ILK-R211A were made using the Quickchange site-directed mutagenesis kit per the manufacturer's instructions (Stratagene Inc.). Adenoviruses encoding TAM67 and JNK1 were purchased from Vector Biolabs and Cell Biolabs, respectively. Infection was conducted in serum-free DMEM, at the multiplicity of infection indicated in the figure legends, for 2 hours. ILK-targeted oligonucleotide siRNA (sequence, 5'-GGGCAAUGACAUUGUCGUG-3') or nontargeted oligonucleotide siRNA (control siRNA no. 1) were purchased from Ambion and used at $20 \mathrm{nM}$ for all cell lines except Rh4 (40 nM). ILK, JNK1, JNK2, and control siRNA-targeted oligonucleotide pools were purchased from Dharmacon and used at $50 \mathrm{nM}$. PAX3-FKHR cloned into pCDNA3.1 was a gift of F. Barr (University of Pennsylvania, Philadelphia, Pennsylvania, USA). SP600125 and the negative control inhibitor $\mathrm{N}^{1}$-Methyl-1,9-pyrazoloanthrone were purchased from Calbiochem (EMD Biosciences) and used at $20 \mu \mathrm{M}$. Transfections were completed with Lipofectamine 2000 (Invitrogen) per the manufacturer's protocol. RD stable clones were created by transfecting $1 \mu \mathrm{g}$ purified plasmid into $2 \times 10^{5} \mathrm{RD}$ cells, selected with $750 \mu \mathrm{g} / \mathrm{ml} \mathrm{G} 418$ and propagated in $500 \mu \mathrm{g} / \mathrm{ml} \mathrm{G} 418$ (Invitrogen).

RNA preparation and real-time PCR. RNA was extracted with TRIzOL (Invitrogen), treated with DNase I (GenHunter Corp.), and purified with the RNeasy kit (Qiagen Inc.) per the manufacturers' instructions. Skeletal muscle RNA was purchased from Clontech Laboratories. RNA $(1 \mu \mathrm{g})$ was reverse transcribed using Superscript II reverse transcriptase (Invitrogen) by random priming. cDNA $(0.5 \mu \mathrm{l})$ was used for real-time PCR in triplicate using SYBR green master mix on a Lightcycler 480 (Roche). Reactions were incubated for 5 minutes at $95^{\circ} \mathrm{C}$, followed by 50 cycles of $95^{\circ} \mathrm{C}$ for 15 seconds, $60^{\circ} \mathrm{C}$ for 40 seconds, and $72^{\circ} \mathrm{C}$ for 15 seconds. Product specificity was confirmed by melt-point analysis. Data was analyzed by the $\Delta \Delta \mathrm{Ct}$ method (44) using 2 housekeeping controls, TATA box-binding protein $(T B P)$ and hypoxanthine phosphoribosyltransferase 1 (HPRT). Primer sequences are detailed in Supplemental Methods.

Western blotting. Protein samples were prepared in EBC lysis buffer $(120$ $\mathrm{mM} \mathrm{NaCl}, 50 \mathrm{mM}$ Tris base, and $0.5 \% \mathrm{NP}-40$ ) supplemented with protease 
and phosphatase inhibitors (Roche Applied Science). Equal amounts of protein were separated by $12 \%$ SDS-PAGE, transferred to Immobilon PVDF membranes (Millipore), and probed with primary antibodies in $10 \mathrm{mM}$ TBS-T. Membranes were probed with infrared dye-tagged secondary antibodies (Li-Cor Biosciences) for 30 minutes and visualized on the Li-Cor Odyssey System (Li-Cor Biosciences). Primary anti-ILK, phospho-Ser9GSK3 $\beta$, phospho-Ser63-c-Jun, phospho-Thr183/Tyr185-JNK, and total JNK antibodies were purchased from Cell Signaling Technology. Anti-GFP and total c-Jun antibodies were purchased from Santa Cruz Biotechnology Inc. Anti-vinculin antibody was purchased from Upstate Biotechnology. Anti-PAX3 antibody was purchased from Abcam Inc. Band quantitation was performed with NIH ImageJ (http://rsbweb.nih.gov/ij/) with phosphoproteins normalized against their respective total proteins and vinculin.

Kinase assay. Protein $(200 \mu \mathrm{g})$ was precleared from lysates with protein Gplus agarose beads and rabbit IgG (Santa Cruz Biotechnology Inc.). Supernatants were immunoprecipitated overnight with protein $\mathrm{G}$-plus agarose beads plus either control rabbit IgG or rabbit monoclonal anti-ILK antibody (Cell Signaling Technologies). Following washes in EBC buffer, 50\% of the pellet was resuspended in $6 \times$ SDS-PAGE sample buffer to represent the immunoprecipitation. The other $50 \%$ was incubated in $40 \mu \mathrm{l}$ of $1 \times$ kinase assay buffer (Cell Signaling) supplemented with $0.25 \mathrm{mM}$ ATP (Fermentas Inc.), with or without $2.5 \mu$ g recombinant GST-tagged GSK3 $\beta$ peptide (Cell Signaling Technology) for 30 minutes at $30^{\circ} \mathrm{C}$. Kinase reactions were terminated by boiling in $6 \times$ SDS-PAGE sample buffer. Samples were resolved by SDS-PAGE and blotted with anti-ILK clone 65.1.9 (Upstate Biotechnology), anti-GST (Millipore), and phospho-Ser9-GSK3 $\beta$ (Cell Signaling) antibodies.

Murine xenografts. Animal experiments were approved by the Hospital for Sick Children animal research ethics board (REB; no. 7372). RD or Rh4 RMS cells $\left(5 \times 10^{6}\right.$ or $1 \times 10^{7}$ cells, respectively) treated with lipofectamine alone or with $I L K$ or control siRNAs were subcutaneously implanted into the right flanks of 6- to 8-week-old female Balb/c nu/nu mice (Charles River Laboratories). Cells $(50 \mu \mathrm{l})$ were coinjected admixed with $50 \mu \mathrm{l}$ certified LDEV-free Matrigel (BD Biosciences). Tumor plugs were monitored daily using calipers, and volume was estimated as $\left(l \times w^{2} \times \pi\right) / 6$, where $l$ is length and $w$ is width. Tumors were left without further manipulation until sacrifice at days $1-3$ or day 32 (ARMS) or 47 (ERMS) after implantation, at which point tumors exceeded $1,000 \mathrm{~mm}^{3}$ or the animals were moribund. Tissues were fixed in $5 \%$ neutral-buffered formalin for 24 hours or snapfrozen in liquid nitrogen and stored at $-80^{\circ} \mathrm{C}$ for further analysis.

Immunohistochemistry. Sections of primary human tumors and xenografts were cut at $4 \mu \mathrm{m}$ and placed onto silane-coated slides. These and preconstructed tissue microarrays were dewaxed and subjected to heat-induced epitope retrieval before blocking for endogenous peroxidase and biotin. Details of antibodies for immunohistochemistry, TUNEL procedures, and Factor VIII scoring are provided in Supplemental Methods. Scoring for ILK and phosphorylated JNK (Thr183/Tyr185) was completed using a modification of a previously described system (45). The immunohistochemical score was reported as the product of the stain intensity and distribution, both with a maximum of 3 , to yield a maximum product of 9 .

Proliferation, colony, and soft agar assays. We conducted 3-(4,5-dimethylthiazol-2-yl)-2,5-diphenyltetrazolium bromide (MTT), colony, and soft agar assays as described previously (46). For cell counting assays, $5 \times 10^{4}$ cells were plated in triplicate in 6 -well plates. Cells were trypsinized and counted at 72 hours after plating.

Statistics. Data were analyzed using Prism (version 3.0; GraphPad Software Inc.), and images were compiled in Photoshop (version 7.0; Adobe Systems). Results are presented as mean \pm SEM unless otherwise indicated. Statistical analysis was performed with 2-tailed Student's $t$ test or 2-way ANOVA with post-hoc Tukey tests, as appropriate. Kaplan-Meier analyses were performed using the log-rank test. For experiments involving multiple cell lines, statistical significance was determined independently for each cell line. A $P$ value less than 0.05 was considered significant.

\section{Acknowledgments}

We thank Noa Alon, Michael Ho, and Bernard Martin for expertise and assistance and Meredith Irwin (Hospital for Sick Children) and Badia Barakat (Monash University) for critical evaluation of the manuscript. This work was funded in part by the Andrew Mizzoni Cancer Research Fund, the David Wielinga Sarcoma Fund, and Marco's Way Tribute Fund (SickKids Foundation). A.D. Durbin is the recipient of a Canadian Institutes of Health Research MD/PhD studentship and a University of Toronto Open Fellowship.

Received for publication November 3, 2008, and accepted in revised form March 25, 2009.

Address correspondence to: David Malkin, Division of Hematology/Oncology, Hospital for Sick Children, 555 University Avenue, Toronto, Ontario M5G 1X8, Canada. Phone: (416) 813-5348; Fax: (416) 813-5327; E-mail: david.malkin@sickkids.ca. Or to: Gregory E. Hannigan, Centre for Cancer Research, Monash Institute of Medical Research, 246 Clayton Road, Clayton, 3168 Victoria, Australia. Phone: 61-03-9594-7245; Fax: 61-03-9594-7252; E-mail: greg.hannigan@med.monash.edu.au.
1. Breneman, J.C., et al. 2003. Prognostic factors and clinical outcomes in children and adolescents with metastatic rhabdomyosarcoma--a report from the Intergroup Rhabdomyosarcoma Study IV. J. Clin. Oncol. 21:78-84.

2. Wexler, L.H., Crist, W.M., and Helman, L.J. 2002. Rhabdomyosarcoma and the undifferentiated sarcomas. In Principles and practice of pediatric oncology. 4th edition. P.A. Pizzo and D.G. Poplack, editors. Lippincott Williams \& Wilkins. Philadelphia, Pennsylvania, USA. 939-971.

3. Davicioni, E., et al. 2006. Identification of a PAXFKHR gene expression signature that defines molecular classes and determines the prognosis of alveolar rhabdomyosarcomas. Cancer Res. 66:6936-6946.

4. Karnik, P., et al. 1998. Two distinct tumor suppressor loci within chromosome $11 \mathrm{p} 15$ implicated in breast cancer progression and metastasis. Hum. Mol. Genet. 7:895-903.

5. Hannigan, G.E., et al. 1996. Regulation of cell adhesion and anchorage-dependent growth by a new beta 1 -integrin-linked protein kinase. Nature. 379:91-96.

6. Hannigan, G., Troussard, A.A., and Dedhar, S. 2005. Integrin-linked kinase: a cancer therapeutic target unique among its ILK. Nat. Rev. Cancer. 5:51-63.

7. White, D.E., Cardiff, R.D., Dedhar, S., and Muller, W.J. 2001. Mammary epithelial-specific expression of the integrin-linked kinase (ILK) results in the induction of mammary gland hyperplasias and tumors in transgenic mice. Oncogene. 20:7064-7072.

8. Yau, C.Y., Wheeler, J.J., Sutton, K.L., and Hedley, D.W. 2005. Inhibition of integrin-linked kinase by a selective small molecule inhibitor, QLT0254, inhibits the PI3K/PKB/mTOR, Stat3, and FKHR pathways and tumor growth, and enhances gemcitabine-induced apoptosis in human orthotopic primary pancreatic cancer xenografts. Cancer Res. 65:1497-1504

9. Edwards, L.A., et al. 2008. Suppression of VEGF secretion and changes in glioblastoma multiforme microenvironment by inhibition of integrin-linked kinase (ILK). Mol. Cancer Ther. 7:59-70.

10. Tan, C., et al. 2004. Regulation of tumor angiogenesis by integrin-linked kinase (ILK). Cancer Cell. 5:79-90.

11. Lorenz, K., et al. 2007. Integrin-linked kinase is required for epidermal and hair follicle morphogenesis. J. Cell Biol. 177:501-513.

12. Miller, M.G., et al. 2003. Integrin-linked kinase is a positive mediator of L6 myoblast differentiation. Biochem. Biophys. Res. Commun. 310:796-803.

13. Haase, M., et al. 2008. Expression of Integrin-linked Kinase Is Increased in Differentiated Cells. J. Histochem. Cytochem. 56:819-829.

14. Cordes, N. 2004. Overexpression of hyperactive integrin-linked kinase leads to increased cellular radiosensitivity. Cancer Res. 64:5683-5692.

15. Eke, I., et al. 2007. Pharmacological inhibition of EGFR tyrosine kinase affects ILK-mediated cellular radiosensitization in vitro. Int. J. Radiat. Biol. 83:793-802.

16. Hess, F., Estrugo, D., Fischer, A., Belka, C., and 
Cordes, N. 2007. Integrin-linked kinase interacts with caspase- 9 and -8 in an adhesion-dependent manner for promoting radiation-induced apoptosis in human leukemia cells. Oncogene. 26:1372-1384.

17. Chen, P., Shen, W.Z., and Karnik, P. 2004. Suppression of malignant growth of human breast cancer cells by ectopic expression of integrin-linked kinase. Int. J. Cancer. 111:881-891.

18. Shaulian, E., and Karin, M. 2002. AP-1 as a regulator of cell life and death. Nat. Cell Biol. 4:E131-E136.

19. Huang, S., et al. 2003. Sustained activation of the JNK cascade and rapamycin-induced apoptosis are suppressed by p53/p21(Cip1). Mol. Cell. 11:1491-1501.

20. Huang, S., et al. 2004. Inhibition of mammalian target of rapamycin activates apoptosis signal-regulating kinase 1 signaling by suppressing protein phosphatase 5 activity. J. Biol. Chem. 279:36490-36496.

21. Ostrovsky, O., Bengal, E., and Aronheim, A. 2002. Induction of terminal differentiation by the c-Jun dimerization protein JDP2 in C2 myoblasts and rhabdomyosarcoma cells. J. Biol. Chem. 277:40043-40054.

22. Mauro, A., et al. 2002. PKCalpha-mediated ERK, JNK and p38 activation regulates the myogenic program in human rhabdomyosarcoma cells. J. Cell Sci. 115:3587-3599.

23. Perdiguero, E., Ruiz-Bonilla, V., Serrano, A.L., and Munoz-Canoves, P. 2007. Genetic deficiency of p38alpha reveals its critical role in myoblast cell cycle exit: the p38alpha-JNK connection. Cell Cycle. 6:1298-1303

24. Lehtinen, S.K., Rahkila, P., Helenius, M., Korhonen, P., and Salminen, A. 1996. Down-regulation of transcription factors AP-1, Sp-1, and NF-kappa B precedes myocyte differentiation. Biochem. Biophys. Res. Commun. 229:36-43.

25. Quelo, I., Gauthier, C., Hannigan, G.E., Dedhar, S., and St-Arnaud, R. 2004. Integrin-linked kinase regulates the nuclear entry of the c-Jun coactivator alpha-NAC and its coactivation potency. J. Biol. Chem. 279:43893-43899.
26. Tamura, S., et al. 2006. PP2C family members play key roles in regulation of cell survival and apoptosis. Cancer Sci. 97:563-567.

27. Aoyagi, Y., Fujita, N., and Tsuruo, T. 2005. Stabilization of integrin-linked kinase by binding to Hsp90. Biochem. Biophys. Res. Commun. 331:1061-1068.

28. Wan, X., and Helman, L.J. 2003. Levels of PTEN protein modulate Akt phosphorylation on serine 473 , but not on threonine 308 , in IGF-II-overexpressing rhabdomyosarcomas cells. Oncogene. 22:8205-8211.

29. Chung, D.H., et al. 1998. ILK (beta1-integrin-linked protein kinase): a novel immunohistochemical marker for Ewing's sarcoma and primitive neuroectodermal tumour. Virchows Arch. 433:113-117.

30. Persad, S., et al. 2001. Regulation of protein kinase B/Akt-serine 473 phosphorylation by integrinlinked kinase: critical roles for kinase activity and amino acids arginine 211 and serine 343. J. Biol. Chem. 276:27462-27469.

31. Ren, Y.X., et al. 2008. Mouse mesenchymal stem cells expressing PAX-FKHR form alveolar rhabdomyosarcomas by cooperating with secondary mutations. Cancer Res. 68:6587-6597.

32. Delcommenne, M., et al. 1998. Phosphoinositide3-OH kinase-dependent regulation of glycogen synthase kinase 3 and protein kinase B/AKT by the integrin-linked kinase. Proc. Natl. Acad. Sci. U. S. A. 95:11211-11216.

33. Persad, S., et al. 2000. Inhibition of integrin-linked kinase (ILK) suppresses activation of protein kinase B/ $\mathrm{Akt}$ and induces cell cycle arrest and apoptosis of PTEN-mutant prostate cancer cells. Proc. Natl. Acad. Sci. U. S. A. 97:3207-3212.

34. Zhang, Y., et al. 2006. Involvement of integrinlinked kinase in carbon tetrachloride-induced hepatic fibrosis in rats. Hepatology. 44:612-622.

35. Leung-Hagesteijn, C., et al. 2005. Integrin-linked kinase mediates bone morphogenetic protein 7 . dependent renal epithelial cell morphogenesis. Mol.
Cell. Biol. 25:3648-3657.

36. Gupta, S., et al. 1996. Selective interaction of JNK protein kinase isoforms with transcription factors. EMBO J. 15:2760-2770.

37. Baker, S.J., et al. 1989. Chromosome 17 deletions and p53 gene mutations in colorectal carcinomas. Science. 244:217-221.

38. Besson, A., et al. 2007. Discovery of an oncogenic activity in p27Kip1 that causes stem cell expansion and a multiple tumor phenotype. Genes Dev. 21:1731-1746.

39. Yokoyama, A., and Cleary, M.L. 2008. Menin critically links MLL proteins with LEDGF on cancerassociated target genes. Cancer Cell. 14:36-46.

40. de la Iglesia, N., et al. 2008. Identification of a PTEN-regulated STAT3 brain tumor suppressor pathway. Genes Dev. 22:449-462.

41. De Giovanni, C., et al. 1993. Uncoupling of growth inhibition and differentiation in dexamethasonetreated human rhabdomyosarcoma cells. Br. J. Cancer. 67:674-679.

42. Dos Santos, N., et al. 2007. Urokinase-type Plasminogen Activator (uPA) is inhibited with QLT0267 a small molecule targeting Integrin-linked Kinase (ILK). Translational Oncogenomics. 2:67-79.

43. Mongroo, P.S., et al. 2004. Beta-parvin inhibits integrin-linked kinase signaling and is downregulated in breast cancer. Oncogene. 23:8959-8970.

44. Schmittgen, T.D., and Livak, K.J. 2008. Analyzing real-time PCR data by the comparative $\mathrm{C}(\mathrm{T})$ method. Nat. Protoc. 3:1101-1108.

45. Somers, G.R., et al. 2006. Pediatric undifferentiated sarcoma of the soft tissues: a clinicopathologic study. Pediatr. Dev. Pathol. 9:132-142.

46. Greenberg, J.A., Somme, S., Russnes, H.E., Durbin, A.D., and Malkin, D. 2008. The estrogen receptor pathway in rhabdomyosarcoma: a role for estrogen receptor-beta in proliferation and response to the antiestrogen $4^{\prime} \mathrm{OH}$-tamoxifen. Cancer Res. 68:3476-3485. 